Document downloaded from:

http://hdl.handle.net/10251/120694

This paper must be cited as:

Giner Navarro, J.; Martínez Casas, J.; Denia, FD.; Baeza González, LM. (2018). Study of railway curve squeal in the time domain using a high-frequency vehicle/track interaction model. Journal of Sound and Vibration. 431:177-191.

https://doi.org/10.1016/j.jsv.2018.06.004

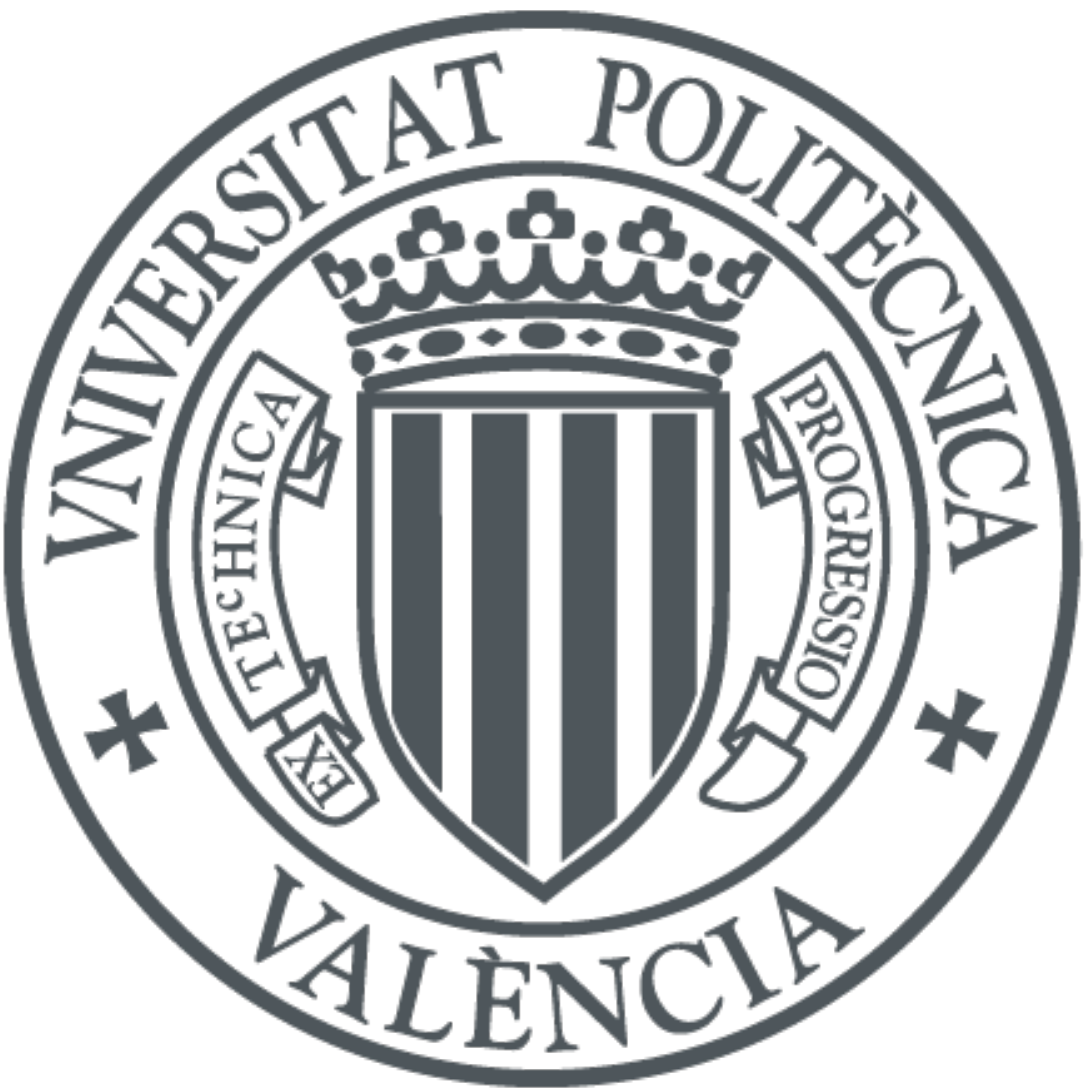

The final publication is available at

http://dx.doi.org/10.1016/j.jsv.2018.06.004

Copyright Elsevier

Additional Information 


\title{
Study of railway curve squeal in the time domain using a high-frequency vehicle/track interaction model
}

\author{
Authors: J. Giner-Navarro ${ }^{1}$, J. Martínez-Casas ${ }^{1}$, F. D. Denia ${ }^{1}$ and L. Baeza ${ }^{2 *}$
}

\begin{abstract}
Affiliation:
${ }^{1}$ Centro de Investigación en Ingeniería Mecánica, Universitat Politècnica de València, Camino de Vera s/n, 46022 Valencia, Spain.

${ }^{2}$ Institute of Sound and Vibration Research, University of Southampton, Southampton SO17 1BJ, UK.

* E-mail corresponding author: L.Baeza@soton.ac.uk
\end{abstract}

Short title: Study of curve squeal in the time domain

\section{Address for correspondence:}

Prof. Luis Baeza

Institute of Sound and Vibration Research

University of Southampton - Highfield

Building 13 / Room 3075

Southampton

SO17 1BJ

United Kingdom

Telephone: (023) 80592798

Facsimile: (023) 80593190

Email: L.Baeza@soton.ac.uk 


\begin{abstract}
:
Railway curve squeal is an intense tonal and annoying type of noise commonly attributed to self-excited vibrations during curving. The mechanisms for its generation remain unclear and it is still a subject of discussion among researchers. Most of them have considered the falling behaviour of the friction coefficient with the slip velocity essential for reenergising the system. Recently, some authors have found that squeal can also appear even for constant friction coefficient through the wheel modal coupling between the normal and tangential directions caused by the wheel/rail contact. This paper particularly evaluates whether the latter mechanism is sufficient to find squeal in curving conditions.
\end{abstract}

The introduction of flexibility in the railway subsystems is required to widen the domain to the high-frequency range in which squeal occurs. One single flexible and rotatory wheelset is considered and suitable forces are prescribed at the primary suspension seats in the current investigation. The rails are modelled through the Moving Element Method (MEM), permitting to extend the range of validity of beam models usually utilised in the literature. This work extends the formulation to rails supported by a viscoelastic Winkler bedding. Both wheelset and track models are coupled by means of a non-linear and unsteady wheel/rail contact model based on Kalker's Variational Theory. Simulation results for different track curvatures and friction coefficients are presented and discussed, showing tonal peaks in the tangential contact forces of the inner wheel. These results can be associated with squeal according to the characterisation of this phenomenon, indicating that squeal can be found in curving conditions using advanced dynamic interaction models even with constant friction coefficient.

\title{
Keywords:
}

Curve squeal; wheel/rail contact; rotating wheelset; Moving Element Method; railway high-frequency dynamics. 


\section{INTRODUCTION.}

Noise appears as one of the most important environmental drawbacks of the railway transportation [1]. Railway tracks are frequently located in the vicinity of urban zones, thus being a source of considerable annoyance for residents [2]. Three categories of wheel/rail noise can be distinguished: rolling noise, impact noise and squeal noise [3]. The present work is focused on the latter, known as curve squeal when the train negotiates sharp curves. This phenomenon is characterised by an intense tonal noise generated in the most sensitive human ear frequency band between 2 and $8 \mathrm{kHz}$ [4], and sometimes even up to $10 \mathrm{kHz}$ according to field measurements in metro and tramway systems [5].

The likelihood of squeal occurrence widely differs on apparently similar conditions and the physical mechanisms responsible for this phenomenon remain unclear. The falling friction mechanism proposed by Rudd [6] has been the most accepted instability mechanism to explain squeal as self-excited vibrations. Rudd also identified three possible excitation mechanisms due to stick/slip cycles in the contact region: (1) lateral creepage at the contact between the wheel tread and the top of the railhead, (2) wheel flange rubbing on the rail gauge face, and (3) longitudinal creepage at the contact on the wheel tread due to differential slip. The first one, closely linked to the curving behaviour of the vehicle, has received the major attention in the literature.

Some recent works $[7,8]$ presented a mechanism called modal coupling through which it is possible to reproduce the curve squeal even with a constant friction coefficient. Although the latter friction coefficient decays with slip velocity, the negative slope of the creep curves (creepage vs. total transmitted forces) in the transversal directions (crucial for squeal $[4,8]$ ) can be considered negligible. This assumption is supported by experimental tests [9] favourably contrasted in a recent work on contact mechanics [10], which concluded that the difference between the maximum and the saturated tangential creep forces shown in standard curves due 
to falling friction is clearly overrated. This lesser influence leads to adopt a constant- $\mu$ hypothesis for dynamic simulations.

The present work develops an advanced vehicle/track interaction model that adopts a non-steady state wheel/rail contact model with a constant friction coefficient. The flexibility of the solids is implemented in order to extend the interaction model to the high-frequency domain in which squeal occurs. The vehicle model is simplified through one single flexible wheelset and the corresponding primary suspension instead of one complete vehicle [11]. In order to implement the effect of the vehicle dynamics, a multibody model for the complete vehicle negotiating a constant radius curve is used to calculate the forces in the primary suspensions, which are implemented as external forces in the wheelset model. The wheelset model accounts the flexibility and the inertial effects associated with its rotation. Since the angle of attack and the creepages are more demanding for the leading wheelset when negotiating a curve, this is more prone to squeal than the rear ones and thus the leading wheelset is the one selected for the simulation. Section 2 of this paper summarises the wheelset model adopted in the present work.

This paper develops a model of the railway track that permits to study the track dynamics extending the frequency range up to $9 \mathrm{kHz}$, covering the frequency range where squeal takes place. The track model consists of two solid rails supported on a Winkler foundation. The Moving Element Method [12] has been adopted in this work by implementing cyclic boundary conditions and a viscoelastic Winkler foundation. The technique and its benefits are presented in Section 3.

The wheelset and the track models are coupled by means of a wheel/rail contact theory that adopts an incremental algorithm described in Section 4. The wheel/rail contact position is obtained from a low frequency commercial package that provides the whole vehicle position on the curve, and a non-linear and non-steady state wheel/rail contact model based on Kalker's Variational Theory [13] is implemented by the authors for obtaining the contact forces. Section 
5 presents simulation results of the squeal phenomenon for different curve radius and friction coefficients. The paper closes with conclusive remarks in Section 6.

\section{VEHICLE MODEL.}

A flexible rotatory wheelset model negotiating a curved track was previously developed in Ref. [11]. In order to model the wheelset travelling on a curved track, two reference frames are considered: a frame $\mathbf{X}_{0} \mathbf{Y}_{0} \mathbf{Z}_{0}$ fixed at an arbitrary point and a trajectory coordinate frame $\mathbf{X}_{\mathrm{T}} \mathbf{Y}_{\mathrm{T}} \mathbf{Z}_{\mathrm{T}}$ that follows the motion of the wheelset on the track (see Fig. 1). The system $\mathbf{X}_{\mathrm{T}} \mathbf{Y}_{\mathrm{T}} \mathbf{Z}_{\mathrm{T}}$ is centred in the undeformed configuration of the wheelset, with the $\mathbf{X}_{\mathrm{T}}$-axis parallel to the forward speed, the $\mathbf{Y}_{\mathrm{T}}$-axis parallel to wheelset axis and the $\mathbf{Z}_{\mathrm{T}}$-axis pointing upwards.

Eulerian coordinates are defined from the trajectory reference frame, through which the properties of the spatial points of the non-deformed configuration domain are obtained. These coordinates do not follow the material points of the solid, nonetheless they are associated with spatial points. Due to the axisymmetric geometry of the wheelset, this methodology permits to represent the displacements in the spatial points from the non-deformed configuration using the vibration modes as basis in the trajectory reference frame, which is:

$$
\mathbf{r}=\boldsymbol{\Phi} \mathbf{q}
$$

being $\mathbf{r}$ the vector that contains the displacements from the undeformed to the deformed configuration, $\boldsymbol{\Phi}$ is the modal matrix computed through a FE model for the frame $\mathbf{X}_{\mathrm{T}} \mathbf{Y}_{\mathrm{T}} \mathbf{Z}_{\mathrm{T}}$, and q contains the modal coordinates. The resulting modal equation of motion for the selected wheelset is [11]:

$$
\ddot{\mathbf{q}}+(\Omega \mathbf{A}+\mathbf{B}) \dot{\mathbf{q}}+\left(\Omega^{2} \mathbf{D}+\Omega \mathbf{E}+\mathbf{K}\right) \mathbf{q}=\boldsymbol{\alpha}+\Omega \boldsymbol{\beta}+\Omega^{2} \boldsymbol{\gamma}+\mathbf{Q}_{c}+\mathbf{Q}_{s}
$$


where $\Omega$ is the angular velocity of the wheelset (the wheelset speed divided by the wheel radius); the matrices $\mathbf{A}, \mathbf{B}, \mathbf{D}, \mathbf{E}$, and vectors $\boldsymbol{\alpha}, \boldsymbol{\beta}$ and $\boldsymbol{\gamma}$ account for the inertial effects associated with the wheelset rotation around the curve and of itself; $\mathbf{K}$ is the modal stiffness matrix (diagonal matrix with the square of the natural frequencies); finally, $\mathbf{Q}_{c}$ and $\mathbf{Q}_{s}$ are the vectors of the generalised forces acting on the flexible wheelset resulting respectively from the wheel/rail contact forces and the forces applied through the primary suspension. The previous matrices are independent of time if the radius of the curve remains constant. A complete description of the wheelset formulation can be found in Ref. [11].

\section{TRACK MODEL.}

The track model consists of two solid rails supported by a viscoelastic Winkler foundation. It is used a reference frame that is attached to the contact patch, hence moving with the vehicle. The associated coordinate vectors point spatial positions while the mass of the rail flows through the fixed spatial mesh with the vehicle speed $V$, which is considered constant along the simulation. A cyclic approach is also followed, introducing cyclic boundary conditions at the ends of the model (the displacements at both ends of the track coincide) that can be interpreted as an infinite track negotiated by an infinite set of identical vehicles uniformly distributed in such a way that each vehicle is set at a constant distance $L$ apart from the adjacent ones. Due to the periodicity of the structure and the loading conditions, the study is reduced to a single section having finite length $L$, whose value is set large enough to avoid interaction between the vehicles. This interaction appears as reflection waves in the receptance function of the rail between $500 \mathrm{~Hz}$ and $2 \mathrm{kHz}$, which are mitigated when increasing the length; from $40 \mathrm{~m}$, those can be considered negligible [14]. The method allows positioning the wheel/rail contact area at a fixed railhead element avoiding the vehicle exceeding the 'downstream' boundary ends since; 
it also permits to implement a mesh with greater refinement around this region, where forces and displacements are higher, contributing to reduce the computational cost.

\subsection{Rail model.}

Fig. 2 presents a 3D track resulting from the extrusion of a UIC60 profile. An inertial coordinate system $\mathbf{X Y Z}$ is defined, with the $\mathbf{X}$-axis parallel to the rail extrusion direction, the $\mathbf{X Y}$-plane parallel to the rail base and the $\mathbf{Z}$-axis pointing upwards. The rail material is supposed to flow with velocity $V$ opposite to the $\mathbf{X}$ direction (as can be seen in Fig. 2). An Eulerian position vector $\mathbf{u}$, associated with the coordinate system, defines the fixed position of a spatial point of the mesh (undeformed configuration). Vector $\mathbf{w}=\mathbf{w}(\mathbf{u}, t)$ is the displacement of a material point that occupies the position $\mathbf{u}$ at the instant $t$ with respect to the undeformed configuration. The position vector $\mathbf{R}$ of the material point is hence

$$
\mathbf{R}=\mathbf{u}+\mathbf{w}(\mathbf{u}, t)
$$

Cyclic boundary conditions set same displacements and derivatives at the model edges of the finite rail. The velocity and acceleration of the material point are computed through the material derivative as follows

$$
\begin{gathered}
\mathbf{v}=\frac{\mathrm{D} \mathbf{R}}{\mathrm{D} t}=\frac{\partial \mathbf{R}}{\partial t}-V \frac{\partial \mathbf{R}}{\partial x}=(-V, 0,0)^{\mathrm{T}}+\dot{\mathbf{w}}-V \frac{\partial \mathbf{w}}{\partial x} \\
\mathbf{a}=\frac{\mathrm{D} \mathbf{v}}{\mathrm{D} t}=\frac{\partial \mathbf{v}}{\partial t}-V \frac{\partial \mathbf{v}}{\partial x}=\ddot{\mathbf{w}}-2 V \frac{\partial \dot{\mathbf{w}}}{\partial x}+V^{2} \frac{\partial^{2} \mathbf{w}}{\partial x^{2}}
\end{gathered}
$$

where $\mathrm{D} / \mathrm{D} t=\partial / \partial t-V \partial / \partial x_{1}$ refers to the material (or total) derivative. The virtual work associated with the inertial forces is evaluated through Eq. (5): 


$$
\delta W=\int_{V o l} \rho \delta \mathbf{w}^{\mathrm{T}} \mathbf{a} \mathrm{d} v=\int_{V o l} \rho \delta \mathbf{w}^{\mathrm{T}} \ddot{\mathbf{w}} \mathrm{d} v-2 V \int_{V o l} \rho \delta \mathbf{w}^{\mathrm{T}} \frac{\partial \dot{\mathbf{w}}}{\partial x} \mathrm{~d} v+V^{2} \int_{V o l} \rho \delta \mathbf{w}^{\mathrm{T}} \frac{\partial^{2} \mathbf{w}}{\partial x^{2}} \mathrm{~d} v
$$

where $\rho \equiv \rho(x, y, z)$ is the density of the material (considered constant as the wheelset). Quadratic shape function elements are used for the mesh, the continuity at element interfaces being $\mathrm{C}^{0}$. Consequently, the integration can only be evaluated properly if the maximum order of differentiation is 1 . Note that, as detailed in Section 3.2, page 59 of Ref. [15] as convergence requirement, if the integrand has derivatives up to order $n$, then the interpolation has to guarantee that its $n-1$ derivatives are continuous $\left(\mathrm{C}^{n-1}\right.$ continuity). From this requirement, the virtual work needs to be computed through an integration by parts to obtain lower order derivatives for the third term of Eq. (6):

$$
\int_{V o l} \rho \delta \mathbf{w}^{\mathrm{T}} \frac{\partial^{2} \mathbf{w}}{\partial x^{2}} \mathrm{~d} x \mathrm{~d} y \mathrm{~d} z=\int_{S} \rho \delta \mathbf{w}^{\mathrm{T}} \frac{\partial \mathbf{w}}{\partial x} \mathrm{~d} y \mathrm{~d} z-\int_{V o l} \rho \frac{\partial\left(\delta \mathbf{w}^{\mathrm{T}}\right)}{\partial x} \frac{\partial \mathbf{w}}{\partial x} \mathrm{~d} x \mathrm{~d} y \mathrm{~d} z .
$$

Since the rail length is selected long enough to have negligible displacements at the model edges, the integrand of the surface integral (only computed over the lateral surfaces corresponding to the rail edges) is close to zero. Therefore, the influence of the surface integral can be neglected from a numerical point of view and the convergence of Eq. (7) is guaranteed, resulting as:

$$
\delta W=\int_{V o l} \rho \delta \mathbf{w}^{\mathrm{T}} \ddot{\mathbf{w}} \mathrm{d} v-2 V \int_{V o l} \rho \delta \mathbf{w}^{\mathrm{T}} \frac{\partial \dot{\mathbf{w}}}{\partial x} \mathrm{~d} v-V^{2} \int_{V o l} \rho \frac{\partial\left(\delta \mathbf{w}^{\mathrm{T}}\right)}{\partial x} \frac{\partial \mathbf{w}}{\partial x} \mathrm{~d} v .
$$

FE interpolation is adopted at this point. The displacements in the volume of the $e$-th element $\mathrm{Vol}^{e}$ are computed by means of the shape functions as follows:

$$
\mathbf{w}(\mathbf{u}, t)=\mathbf{N}^{e}(\mathbf{u}) \mathbf{w}^{e}(t) \quad \text { if } \mathbf{u} \in V o l^{e}
$$


$\mathbf{w}^{e}$ being the nodal displacements and $\mathbf{N}^{e}$ the basis (or form) functions matrix. If Eq. (9) is implemented for the $e$-th element in Eq. (8), the following expression is obtained:

$$
\delta W^{e}=\delta \mathbf{w}^{e^{\mathrm{T}}}\left(\int_{V o l^{e}} \rho \mathbf{N}^{e^{\mathrm{T}}} \mathbf{N}^{e} \mathrm{~d} v \ddot{\mathbf{w}}^{e}-2 V \int_{V o l^{e}} \rho \mathbf{N}^{e^{\mathrm{T}}} \frac{\partial \mathbf{N}^{e}}{\partial x} \mathrm{~d} v \dot{\mathbf{w}}^{e}-V^{2} \int_{V o l^{e}} \rho \frac{\partial \mathbf{N}^{e^{\mathrm{T}}}}{\partial x} \frac{\partial \mathbf{N}^{e}}{\partial x} \mathrm{~d} v \mathbf{w}^{e}\right)
$$

The last expression can be compacted in the following formula:

$$
\delta W^{e}=\delta \mathbf{w}^{e^{\mathrm{T}}}\left(\mathbf{M}^{e} \ddot{\mathbf{w}}^{e}-V \tilde{\mathbf{M}}^{e} \dot{\mathbf{w}}^{e}-V^{2} \hat{\mathbf{M}}^{e} \mathbf{w}^{e}\right)
$$

Following the FEM assembling technique for calculating the global matrices from the element matrices, the equation of motion of the rail is obtained:

$$
\mathbf{M} \ddot{\mathbf{w}}^{F E}-V \tilde{\mathbf{M}} \dot{\mathbf{w}}^{F E}-V^{2} \hat{\mathbf{M}} \mathbf{w}^{F E}=\mathbf{F}_{c}+\mathbf{F}_{W}+\mathbf{F}_{K},
$$

being $\mathbf{w}^{F E}$ one vector that contains the nodal displacements of the whole FE mesh; $\mathbf{M}, \tilde{\mathbf{M}}$ and $\hat{\mathbf{M}}$ are the global matrices that are obtained from the element matrices $\mathbf{M}^{e}, \widetilde{\mathbf{M}}^{e}$ and $\hat{\mathbf{M}}^{e}$; and $\mathbf{F}_{c}, \mathbf{F}_{W}$ and $\mathbf{F}_{K}$ are the generalised force vectors associated with the wheel/rail contact, Winkler foundation and elastic forces, respectively. It must be highlighted that $\mathbf{F}_{K}=-\mathbf{K} \mathbf{w}^{F E}$, and $\mathbf{M}$ and $\mathbf{K}$ are the standard FEM mass and stiffness matrices.

\subsection{Rail support model.}

A continuous support is modelled under the rail through a viscoelastic Winkler foundation with a uniform distribution of vertical stiffness and damping equivalent to discrete rail supports (railpads + ballast). The dynamics associated with the model is similar compared to the beam resting on discrete supports in the high-frequency band [14] in which squeal phenomenon takes place. In this section, the generalised force associated with the Winkler foundation $\mathbf{F}_{W}$ is 
obtained through the formulation of the virtual work for the elastic and viscous forces. The virtual work of the Winkler forces acting on the $e$-th element located on the bottom surface of the rail is:

$$
\delta W_{W}^{e}=-\int_{S^{e}} \delta \mathbf{w}^{\mathrm{T}} \mathbf{k}_{W} \mathbf{w} \mathrm{d} S-\int_{S^{e}} \delta \mathbf{w}^{\mathrm{T}} \mathbf{c}_{W} \frac{\mathrm{D} \mathbf{w}}{\mathrm{D} t} \mathrm{~d} S
$$

being $S^{e}$ the botton surface of the $e$-th element, and $\mathbf{k}_{W}$ and $\mathbf{c}_{W}$ are $3 \times 3$ diagonal matrices that include respectively the stiffness and damping per unit surface in the $\mathbf{X}, \mathbf{Y}$ and $\mathbf{Z}$ directions. By expanding the material derivative, it is obtained:

$$
\delta W_{W}^{e}=-\int_{S^{e}} \delta \mathbf{w}^{\mathrm{T}} \mathbf{k}_{W} \mathbf{w} \mathrm{d} S-\int_{S^{e}} \delta \mathbf{w}^{\mathrm{T}} \mathbf{c}_{W} \dot{\mathbf{w}} \mathrm{d} S+V \int_{S^{e}} \delta \mathbf{w}^{\mathrm{T}} \mathbf{c}_{W} \frac{\partial \mathbf{w}}{\partial x} \mathrm{~d} S .
$$

If the basis presented in Eq. (9) is implemented in the last equation, the following expression is found:

$$
\delta W_{W}^{e}=-\delta \mathbf{w}^{e^{\mathrm{T}}} \int_{S^{e}} \mathbf{N}^{e^{\mathrm{T}}} \mathbf{k}_{W} \mathbf{N}^{e} \mathrm{~d} S \mathbf{w}^{e}-\delta \mathbf{w}^{e^{\mathrm{T}}} \int_{S^{e}} \mathbf{N}^{e^{\mathrm{T}}} \mathbf{c}_{W} \mathbf{N}^{e} \mathrm{~d} S \dot{\mathbf{w}}^{e}+V \delta \mathbf{w}^{e^{\mathrm{T}}} \int_{S^{e}} \mathbf{N}^{e^{\mathrm{T}}} \mathbf{c}_{W} \frac{\partial \mathbf{N}^{e}}{\partial x} \mathrm{~d} S \mathbf{w}^{e}
$$

Following the same procedure than the one in Section 3.1, the expression of the generalised force is

$$
\mathbf{F}_{W}=-\mathbf{K}_{W} \mathbf{w}^{F E}-\mathbf{C}_{W} \dot{\mathbf{w}}^{F E}+V \widetilde{\mathbf{C}}_{W} \mathbf{w}^{F E},
$$

where the global matrices $\mathbf{K}_{W}, \mathbf{C}_{W}$ and $\widetilde{\mathbf{C}}_{W}$ are assembled from the element matrices $\mathbf{K}_{W}^{e}$, $\mathbf{C}_{W}^{e}$ and $\widetilde{\mathbf{C}}_{W}^{e}$, and deduced from Eq. (15):

$$
\begin{gathered}
\mathbf{K}_{W}^{e}=\int_{S^{e}} \mathbf{N}^{e^{\mathrm{T}}} \mathbf{k}_{W} \mathbf{N}^{e} \mathrm{~d} S, \\
\mathbf{C}_{W}^{e}=\int_{S^{e}} \mathbf{N}^{e^{\mathrm{T}}} \mathbf{c}_{W} \mathbf{N}^{e} \mathrm{~d} S,
\end{gathered}
$$




$$
\widetilde{\mathbf{C}}_{W}^{e}=\int_{S^{e}} \mathbf{N}^{e^{\mathrm{T}}} \mathbf{c}_{W} \frac{\partial \mathbf{N}^{e}}{\partial x} \mathrm{~d} S .
$$

\subsection{Eulerian modal approach.}

By combining Eqs. (12) and (16), the equation motion of the rail on a Winkler foundation is:

$$
\mathbf{M} \ddot{\mathbf{w}}^{F E}+\left(\mathbf{C}_{W}-V \tilde{\mathbf{M}}\right) \dot{\mathbf{w}}^{F E}+\left(\mathbf{K}+\mathbf{K}_{W}-V \widetilde{\mathbf{C}}_{W}-V^{2} \hat{\mathbf{M}}\right) \mathbf{w}^{F E}=\mathbf{F}_{c}
$$

In order to reduce the dimension of the problem, the mass-normalised mode shapes of the undamped rail are adopted as basis of the rail displacements. Consequently, the FE nodal displacements are obtained from the modal matrix $\boldsymbol{\theta}$ as follows:

$$
\mathbf{w}^{F E}=\boldsymbol{\theta} \mathbf{p},
$$

being $\mathbf{p}$ the modal coordinates. The modal matrix verifies:

$$
\boldsymbol{\theta}^{\mathrm{T}}\left(\mathbf{K}+\mathbf{K}_{W}\right) \boldsymbol{\theta}=\operatorname{diag}\left(\omega_{r}^{2}\right)=\left[\begin{array}{lll}
\ddots & & 0 \\
& \omega_{r}^{2} & \\
0 & & \ddots
\end{array}\right],
$$

being $\omega_{r}$ the undamped natural frequencies, and

$$
\boldsymbol{\theta}^{\mathrm{T}} \mathbf{M} \boldsymbol{\theta}=\mathbf{I},
$$

where $\mathbf{I}$ is the unit matrix. The equation of motion for the rail through this modal approach is

$$
\ddot{\mathbf{p}}+\boldsymbol{\theta}^{\mathrm{T}}\left(\mathbf{C}_{W}-V \tilde{\mathbf{M}}\right) \boldsymbol{\theta} \dot{\mathbf{p}}+\left[\operatorname{diag}\left(\omega_{r}^{2}\right)-\boldsymbol{\theta}^{\mathrm{T}}\left(V \widetilde{\mathbf{C}}_{W}+V^{2} \hat{\mathbf{M}}\right) \boldsymbol{\theta}\right] \mathbf{p}=\boldsymbol{\theta}^{\mathrm{T}} \mathbf{F}_{c}
$$




\section{MODEL OF WHEEL/RAIL CONTACT FORCES.}

Eqs. (2) and (24) are coupled through the wheel/rail contact forces in modal coordinates, identified as $\mathbf{Q}_{c}$ and $\boldsymbol{\theta}^{\mathrm{T}} \mathbf{F}_{c}$ for the wheelset and track equations of motion, respectively. The position and velocity of the contact points on the surfaces of both inner and outer wheels and rails are determined in each time step to calculate the relative wheel/rail motion required for the computation of the normal and tangential contact force. This force expressed in Eulerian modal coordinates is applied on the wheel and the rail surfaces at the contact point.

\subsection{Normal contact model.}

The normal contact problem is solved using an incremental approach. For the wheelset and rail profiles, friction and curving conditions considered, the quasi-static solution of the railway interaction model is computed through a pre-processor of a commercial vehicle/track interaction software based on multibody techniques. The software considers elastic contact, permitting to determine a single contact point on each wheel/rail pair assuming both wheel and rail undeformable and computing the relative lateral displacement of the wheel on the rail; the quasi-static contact force applied to the contact point is also provided. The lateral displacement will be considered as mean value around which the contact point will oscillate during the numerical integration assuming small variations. The contact displacements associated with the wheel and the rail, $\mathbf{w}_{c}$ and $\mathbf{r}_{c}$, respectively, are calculated through the modal superposition principle.

The wheel/rail incremental distance vector is computed assuming that both surfaces are undeformable:

$$
\Delta=\mathbf{r}_{c}-\mathbf{w}_{c} .
$$


The quasi-static normal contact force permits to estimate the corresponding approach $\bar{\delta}$ through Hertzian theory [16]:

$$
\bar{\delta}=\left(\frac{\overline{F_{3}}}{K_{H}}\right)^{2 / 3}
$$

where $K_{H}$ is a contact stiffness estimated from the material properties and curvatures in the contact point [17], and $\bar{F}_{3}$ is the quasi-static normal contact force. The incremental approach is obtained by projecting the distance $\Delta$ along the direction normal to the contact plane:

$$
\Delta \delta=\Delta^{\mathrm{T}} \mathbf{x}_{3},
$$

where $\mathbf{x}_{3}$ is the unit normal vector. The total normal force in the contact area $F_{3}$ at each time step for the numerical integration can finally be estimated using again Eq. (26) after adding the incremental approach to the quasi-static one:

$$
F_{3}=\bar{F}_{3}+\Delta F_{3}= \begin{cases}K_{H}(\bar{\delta}+\Delta \delta)^{3 / 2} & \text { if }(\bar{\delta}+\Delta \delta)>0 \\ 0 & \text { if }(\bar{\delta}+\Delta \delta) \leq 0\end{cases}
$$

The contact area and the normal traction distribution are obtained by means of the Hertzian contact model [16] from the normal force $F_{3}$.

\subsection{Tangential contact model.}

The tangential contact problem is solved by implementing Kalker's algorithm CONTACT [13]. Again, an incremental approach is adopted assuming small variations of the creepages around the quasi-static longitudinal $\bar{\xi}_{1}$, lateral $\bar{\xi}_{2}$ and spin $\bar{\xi}_{s p}$ values provided by the multibody software:

$$
\xi_{1}=\frac{1}{V} \dot{\Delta}^{\mathrm{T}} \mathbf{x}_{1}+\bar{\xi}_{1}
$$




$$
\begin{gathered}
\xi_{2}=\frac{1}{V} \dot{\Delta}^{\mathrm{T}} \mathbf{x}_{2}+\bar{\xi}_{2} \\
\xi_{s p}=\bar{\xi}_{s p}
\end{gathered}
$$

where $\mathbf{x}_{1}$ and $\mathbf{x}_{2}$ are unit vectors in the rolling and lateral direction, respectively. Following the non-steady CONTACT algorithm, the computation of the tangential traction distribution also depends on the displacements produced in the present mesh by the computed traction in the previous instant of the numerical integration. The longitudinal and lateral contact forces, $F_{1}$ and $F_{2}$ respectively, are estimated and, together with the normal one $F_{3}$, projected along the trajectory frame $\mathbf{X}_{\mathbf{T}} \mathbf{Y}_{\mathbf{T}} \mathbf{Z}_{\mathbf{T}}$ for the wheelset and the inertial coordinate system $\mathbf{X Y Z}$ for the track, accounting for the inclination of the wheel/rail contact plane. Finally, the resulting projections are applied in both wheels and the rails (with opposite sign) in the corresponding contact points as external actions, providing the generalised force vectors in modal coordinates associated with the contact forces, $\mathbf{Q}_{c}$ and $\boldsymbol{\theta}^{\mathrm{T}} \mathbf{F}_{c}$, included in Eqs. (2) and (24), respectively.

\section{SIMULATION RESULTS.}

The formulations described in Sections 2, 3 and 4 are implemented in a complete high-frequency wheelset/track interaction model. This section is divided in three subsections: Section 5.1 gathers the parameters used in each simulation in curve computed, Section 5.2 presents the time response of the lateral contact forces and Section 5.3 analyses the corresponding frequency spectrum in order to characterise the curving response of the railway system modelled. 


\subsection{Simulation parameters.}

The leading wheelset selected in this work is meshed with 12340 solid quadratic elements (20-nodes) and includes a total of 260145 degrees of freedom in physical coordinates. It is equipped with a solid axle wheelset in which the wheels corresponding to S1002 profile and the brake discs are meshed as a unique structure. The track is represented by two rails with UIC60 profile inclined 1/40 and $42 \mathrm{~m}$ length are modelled with 8452 solid quadratic elements and include a total of 170175 degrees of freedom each in physical coordinates. Both are supported by a uniform viscoelastic Winkler bedding of $43.7 \mathrm{MN} / \mathrm{m}$ for the vertical stiffness and $12.6 \mathrm{kNs} / \mathrm{m}$ for the vertical damping equivalent to discrete rail supports. According to [18], the stiffness and damping in the longitudinal and lateral directions are modelled as $10 \%$ and $80 \%$ of the previous values, respectively. The mesh is longitudinally refined around the centre of the rail, with a central element length of $1 \mathrm{~cm}$. From the modal approach adopted, 400 vibration modes have been selected for the wheelset and 2000 modes for both rails in order to cover a frequency range up to $9 \mathrm{kHz}$. The time step used in the integration scheme is $5 \times 10^{-6} \mathrm{~s}$ and the total time simulated is $1 \mathrm{~s}$. The spatial resolution in the contact area is 0.25 and $1 \mathrm{~mm}$ in longitudinal and lateral directions, respectively.

It is intended to evaluate the interaction model in curving conditions with a constant friction coefficient in order to see whether the constant coefficient mechanism is sufficient itself to generate instabilities in the contact dynamics that can be potentially associated with curve squeal. Table 1 presents the relevant input data corresponding to eight simulations carried out for two curve radii and four friction coefficients. The vehicle speed $V$ is set for both curve radii to make the non-compensated acceleration zero.

Table 2 gathers the quasi-static solution for the lateral position of the contact point (see Fig. 3) and the creepages for both wheels of the leading wheelset corresponding to each simulation 
case computed through multibody software. The Y-axis points forward the centre of the curve as illustrated in Fig. 1. Table 3 contains the quasi-static conditions of the leading wheelset for Simulation I (chosen for illustrative purposes), with the lateral and vertical displacements of its centre of gravity, the angle of attack and the forces from the carbody. Table 4 lists the lateral variations of the position of the contact point for both wheels and rails (again with respect to the nominal point), the normal contact angle, creepages and creep forces for Simulation I. All these values are used as mean values in the incremental contact algorithm adopted. Fig. 4 shows the location of the wheel/rail contact for the inner and the outer pairs corresponding to Simulation I; the associated normal traction distributions are also presented.

\subsection{Lateral contact response.}

The insertion of the vehicle in a curve directly depends on the curve radius, which defines the angle of attack of the leading wheelset. Since the lateral forces can be estimated from the angle of attack [4], the lateral contact dynamic are closely linked to the conditions that characterise the curve and, hence, to the curve squeal phenomena. The investigation of the lateral contact response appears a good approach to detect the squeal occurrence for the simulation cases run.

Fig. 5 presents the time response corresponding to Simulation I. The amplitude of the lateral oscillations of the inner wheel is shown in Fig. 5(a) while the outer one is depicted in Fig. 5(b). The inner wheel shows an oscillating response that converges around a mean value of $-12.8 \mathrm{kN}$ and high amplitudes around $8.5 \mathrm{kN}$, ten times higher than the outer wheel. Much higher amplitudes for the lateral contact forces in the inner wheel have also been found in the rest of the simulations. These results are in agreement with most of the observations that indicate that highest squeal noise amplitudes are usually generated by the leading inner wheel of a bogie [4]. 
Fig. 6 (zoomed views) compares the tangential contact force and the tangential traction limit defined by the friction coefficient times the normal contact force for Simulation I. The inner wheel (Fig. 6(a)) presents cycles defined by a stick phase when the tangential total force (continuous line) is below the traction bound (dashed line) and a slip phase when both curves overlap. Step 1 marked in Fig. 6(a) corresponds to the stick phase (partial stick) in which the contact area is divided into a stick zone located in the leading edge and a slip zone that surrounds the previous one. Step 2 corresponds to the slip phase in which the contact area is in full slip. For the outer wheel, Fig. 6(b) shows that the tangential force is continuously below the traction bound without reaching full slip at any time. Hence, these stick/slip cycles are only observed in the inner wheel and they can be interpreted as self-induced vibrations in curving conditions that come from the railway dynamics itself.

\subsection{Frequency spectrum.}

The frequency spectrum corresponding to the tangential contact force of the inner wheel is evaluated and shown in Fig. 7. It reveals strongly tonal peaks for particular frequencies in the high-frequency domain that correspond to the expected response of curve squeal phenomenon according to the literature [4-8]. For the simulations carried out, these peaks arise from the complex physical process that governs the contact dynamics throughout the computation. These results confirm that the wheelset/track model implemented in this work is able to reproduce this high-frequency tonal response even with a constant friction coefficient and, hence, this mechanism is revealed as sufficient for the generation of squeal in curving conditions.

The frequencies at which these peaks occur are associated with the oscillating frequencies of the stick/slip and the traction bound (corresponding to the normal contact force) cycles previously visualised, especially if most of the cycle is in full slip. The stick/slip frequencies are 
or close to be multiples of the traction bound frequency. As shown in Fig. 6(a), stick/slip cycles can be observed in which the frequency associated with the normal contact force (estimated in the graph as the inverse of the period - distance between consecutive crests) is about $1.6 \mathrm{kHz}$ and the three oscillations observed in each stick phase lead to a frequency about $4.8 \mathrm{kHz}$ for the tangential force. As a consequence, these frequencies present marked peaks in the frequency spectrum shown in Fig. 7.

Table 5 summarises the frequency peaks corresponding to the lateral contact force for the inner wheel in all the simulations carried out. It is shown that all the cases present a tonal behaviour in the high-frequency domain but with no clear tendencies. For the curve radius of $120 \mathrm{~m}$, the main peaks are found at high frequencies: $7.4 \mathrm{kHz}$ for $\mu=0.20$ and 0.60 , and $4.5 \mathrm{kHz}$ for $\mu=0.32$ and 0.40 . For the curve radius of $500 \mathrm{~m}$, peaks are found however at lower frequencies around $1.6 \mathrm{kHz}$ and the maximum amplitudes are associated with higher friction coefficients. These results suggest that high friction values favour a more pronounced tonal response in curving conditions, which is in agreement with the well-known fact that low friction conditions (wet weather, lubrication) reduce the likelihood of squeal [8]. Anyway, the current parameter study should be extended to establish consistent relationships between squeal characteristics and curve radius and friction coefficient.

The squeal mechanism for constant friction has been associated in the literature with the wheel modal coupling from the measurements that found squeal frequencies close to some natural frequencies of the wheel [4]. This coupling is based on at least two degrees of freedom, between which an exchange of energy takes place by damping one of the modes while the other is energised. The previous simulations reveal that preliminary there is not only a single mode that is energised just as simple models predict, but several modes that are energised. Some works that model the wheel as an individual substructure have found that the excited natural 
frequencies of curve squeal correspond to axial wheel modes with zero nodal circles $[19,20]$. Table 6 details the closest wheelset modes associated with the main squeal frequencies gathered in Table 5. Their classification has been made by visualising the deformed configuration associated with each mode considering the torsional, axial and radial contributions from the axle and the brake discs. Only frequencies in bold correspond exactly to a wheelset mode. Axial wheel mode $(2,0, a)$ with two nodal diameters and zero nodal circles corresponds to the main frequency of $1.6 \mathrm{kHz}$ for Simulation I. Axial wheel modes with zero nodal circles $(m=0)$ are associated with squeal frequencies up to $4.5 \mathrm{kHz}$, which agrees with the literature [4,8]. For illustration purposes, Fig. 8 shows the mode shapes associated with the squeal frequencies 1.6 and $4.8 \mathrm{kHz}$, both representing axial wheel modes for the monobloc wheelset with $m=0$ and $m=1$, respectively. Radial wheel modes appear for higher frequencies and axial axle modes also intervene in some unstable frequencies.

The influence of the rotating matrix terms associated with the angular velocity of the wheelset in Eq. (2) is evaluated on the previous simulations in curving conditions. Fig. 9 shows the comparison using a rotatory and non-rotatory formulation for the wheelset in the interaction model, where relevant discrepancies arise in the high-frequency domain for the lateral contact forces. For the curve radius of $500 \mathrm{~m}$ (Simulation II), the non-rotating simulation (dashed line) presents more content in the high-frequency range as seen in Fig. 9(a). The pronounced peaks around 3.8 and $6.1 \mathrm{kHz}$ are substantially mitigated for the rotating case (solid line). Another interesting observation is that the unstable frequencies are slightly shifted to higher values for the rotating case. In the simulation under study, it can be deduced that forward modes especially influence the curving dynamics in the occurrence of squeal. For the curve radius of $120 \mathrm{~m}$ (Simulations III and IV), the frequency content is appreciably higher than the previous one, especially in the range of $1-4 \mathrm{kHz}$ as seen in Figs. 9(b) and (c). The attenuation of the rotating case with respect to the non-rotating one is not so clear in this range, but the frequency shift of 
the rotating peaks to the right can be appreciated. Fig. 9(c) shows that the non-rotating case are strongly attenuated in the range of $7-9 \mathrm{kHz}$.

\section{CONCLUSIONS.}

The present work proposes an advanced non-linear time-domain model to evaluate the railway vehicle/track interaction in curving conditions. The vehicle is confined in the leading wheelset that negotiates a cyclic rail continuously supported by a Winkler bedding. The wheelset model includes the effects associated with the rotation and the curved trajectory and the rail is formulated through an amended version of the Moving Element Method, which permits to fix the contact region in a spatial point of the mesh. Both models are flexible and follow an Eulerian-modal approach in order to keep the computational effort as low as possible. An incremental methodology based on small displacements is implemented for the contact model. The contact conditions are previously estimated from the quasi-static solution given by commercial multibody software and used as initial conditions for the time integration.

Time simulations are run for the wheelset negotiating tight curves in order to evaluate the lateral contact and to investigate curve squeal for constant friction parameters during the curve. Results give only limited insight into the precise underlying mechanism, showing strong unstable lateral contact forces with stick/slip oscillations for the inner wheel. The associated frequency spectrum reveals a strong tonal behaviour in the high-frequency domain. The proposed model is hence able to reproduce the unstable and tonal response that characterises curve squeal even with constant friction.

These results are also in good agreement with some observations reported in the literature about squeal. The unstable frequencies are associated with stick/slip cycles that arise from the non-linearities that govern the contact dynamics $[19,20]$. The unstable peaks decrease their 
amplitudes for low friction values, in line with the fact that low friction conditions reduce the likelihood of squeal [4]. Curve radius (associated with lateral creepage and lateral contact position) and frictional properties are key parameters with significant influence on the intensity of curve squeal $[4,6]$. On other hand, results confirm that the rotation of the wheelset has a crucial influence on curve squeal. The inclusion of the terms associated with the rotation shifts squeal frequencies and it strongly affects the frequency content (amplitude of the tonal peaks) in the high-frequency band, where the precision of the modal approach is less accurate. This band comprises most of the resonances associated with the wheelset, and the gyroscopic effects due to its rotation cause the splitting of these resonances [21]. Further work is needed, focused on a parameter study of these terms in the wheelset equation of motion, to find out why this effect is confined to the high frequency range.

\section{ACKNOWLEDGEMENTS.}

The authors gratefully acknowledge the financial support of Spanish Ministry of Economy, Industry and Competitiveness and the European Regional Development Fund (project TRA2017-84701-R), as well as Generalitat Valenciana (project Prometeo/2016/007) and European Commission through the project "RUN2Rail - Innovative RUNning gear soluTiOns for new dependable, sustainable, intelligent and comfortable RAIL vehicles" (Horizon 2020 Shift2Rail JU call 2017, grant number 777564).

\section{REFERENCES.}

[1] J. M. Fields, J. G. Walker, Comparing the relationships between noise level and annoyance in different surveys: A railway noise vs. aircraft and road traffic comparison, Journal of Sound and Vibration 81 (1) (1982) 51-80. 
[2] U. Moehler, Community response to railway noise: A review of social surveys, Journal of Sound and Vibration 120 (2) (1988) 321-332.

[3] D. J. Thompson, C. J. C. Jones, A review of the modelling of wheel/rail noise generation, Journal of Sound and Vibration 231 (3) (2000) 519-536.

[4] D. J. Thompson, Railway Noise and Vibration: Mechanisms, Modelling and Means of Control, Elsevier, Oxford, UK, 2009.

[5] N. Vicent, J. R. Koch, H. Chollet, J. Y. Guerder, Curve squeal of urban rolling stock - Part 1: State of the art and field measurements, Journal of Sound and Vibration 293 (2006) 691-700.

[6] M. J. Rudd, Wheel/rail noise - Part II: Wheel squeal, Journal of Sound and Vibration 46 (3) (1976) 381-394.

[7] Ch. Glocker, E. Cataldi-Spinola, R. I. Leine, Curve squealing of trains: Measurement, modelling and simulation, Journal of Sound and Vibration 324 (2009) 365-386.

[8] A. Pieringer, A numerical investigation of curve squeal in the case of constant wheel/rail friction, Journal of Sound and Vibration 333 (18) (2014) 4295-4313.

[9] A. Alonso, A. Guiral, L. Baeza, S. D. Iwnicki, Wheel-rail contact: experimental study of the creep forces-creepage relationships, Vehicle System Dynamics 52 (S1) (2014) 469-487.

[10] J. Giner-Navarro, L. Baeza, P. Vila, A. Alonso, Study of falling friction effect on rolling contact parameters, Tribology Letters 65 (2017).

[11] J. Martínez-Casas, E. Di Gialleonardo, S. Bruni, L. Baeza, A comprehensive model of the railway wheelset-track interaction in curves, Journal of Sound and Vibration 333 (2014) $4152-4169$.

[12] C. G. Koh, J. S. Y. Ong, D. K. H. Chua, J. Feng, Moving Element Method for train-track dynamics, International Journal for Numerical Methods in Engineering 56 (2003) 1549-1567. 
[13] J. J. Kalker, Three-dimensional elastic bodies in rolling contact, Kluwer Academic Publishers, Dordrecht, Boston, London, 1990.

[14] J. Giner-Navarro. Advanced techniques for the time-domain modelling of high-frequency train/track interaction, Ph.D. Thesis, Centro de Investigación en Ingeniería Mecánica, Universitat Politècnica de València, València, Spain, 2017.

[15] O. C. Zienkiewicz, R. L. Taylor, J. Z. Zhu, The finite element method: Its basis and fundamentals, Elsevier Butterworth-Heinemann, 2005.

[16] H. Hertz, Ueber die Berührung fester elastischer Körper, Journal für reine und angewandte Mathematik, 92 (1882) 156-171.

[17] A. Pieringer, P.T. Torstensson, J. Giner-Navarro, L. Baeza, Investigation of railway curve squeal using a combination of frequency- and time-domain models, Proceedings of the 12th International Workshop on Railway Noise, Terrigal, Australia (2016) 444-451.

[18] A. Pieringer, P.T. Torstensson, J. Giner-Navarro, Curve squeal of rail vehicles: linear stability analysis and non-linear time-domain simulation, Proceedings of the 3rd International Conference on Railway Technology: Research, Development and Maintenance, Cagliari, Italy (2016) 1759-3433.

[19] D. J. Thompson, C. J. C. Jones, Sound radiation from a vibrating railway wheel, Journal of Sound and Vibration 253 (2) (2002) 401-419.

[20] J. R. Koch, N. Vincent, H. Chollet, O. Chiello, Curve squeal of urban rolling stock - Part 2: Parametric study on a 1/4 scale test rig, Journal of Sound and Vibration 293 (2006) 701-709. 
[21] X. Liu, P. A. Meehan, Investigation of the effect of lateral adhesion and rolling speed on wheel squeal noise, Proceedings of the Institution of Mechanical Engineers, Part F: Journal of Rail and Rapid Transit, Cagliari, Italy 227 (5) (2013) 469-480. 


\section{FIGURE 1}

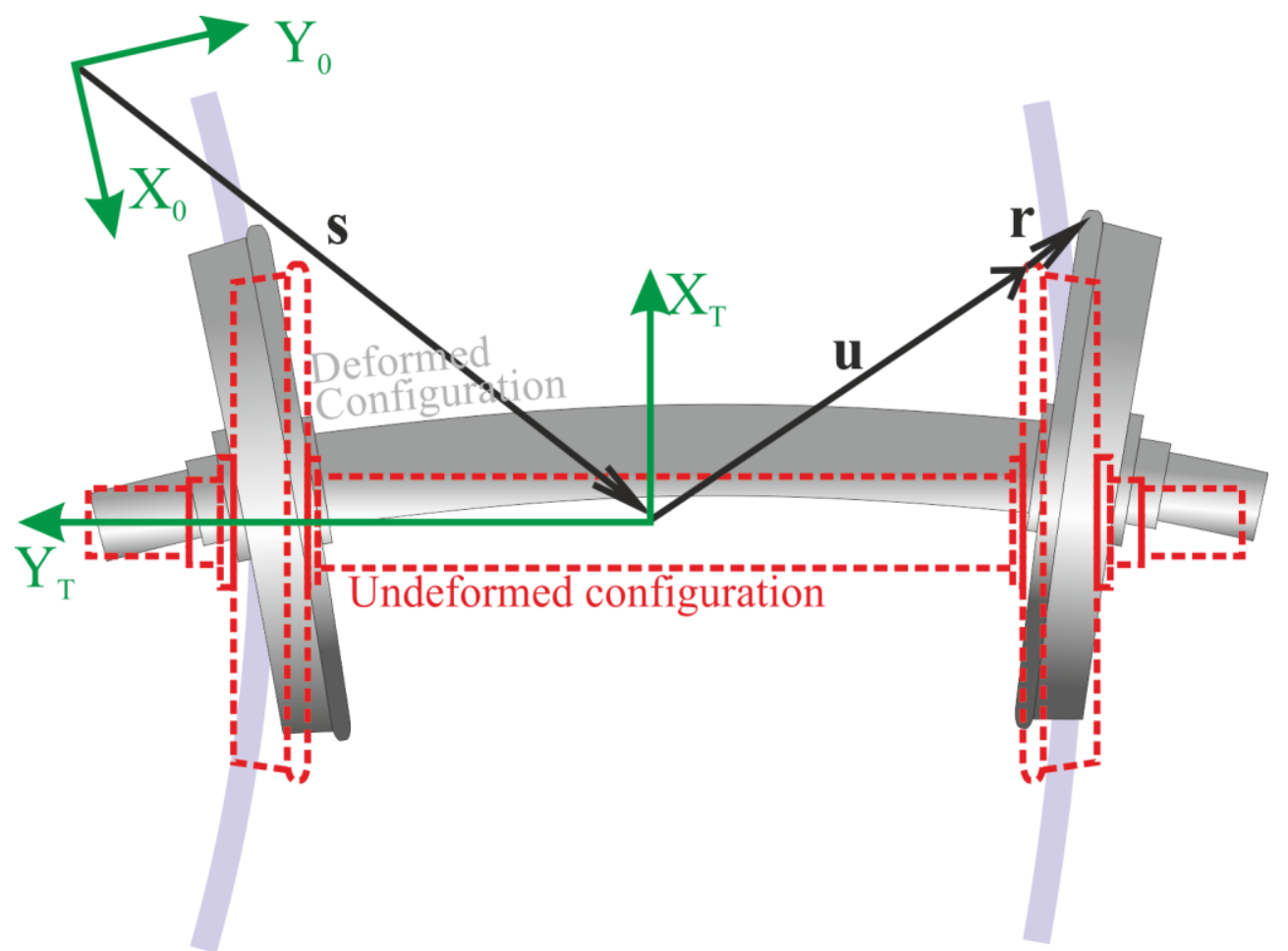

Figure 1: Reference frames and position vectors. The undeformed configuration of the wheelset is shown in dashed trace; a generic position of the flexible wheelset is sketched in solid colours.

Authors: J. Giner-Navarro, J. Martínez-Casas, F. D. Denia and L. Baeza. 


\section{FIGURE 2}

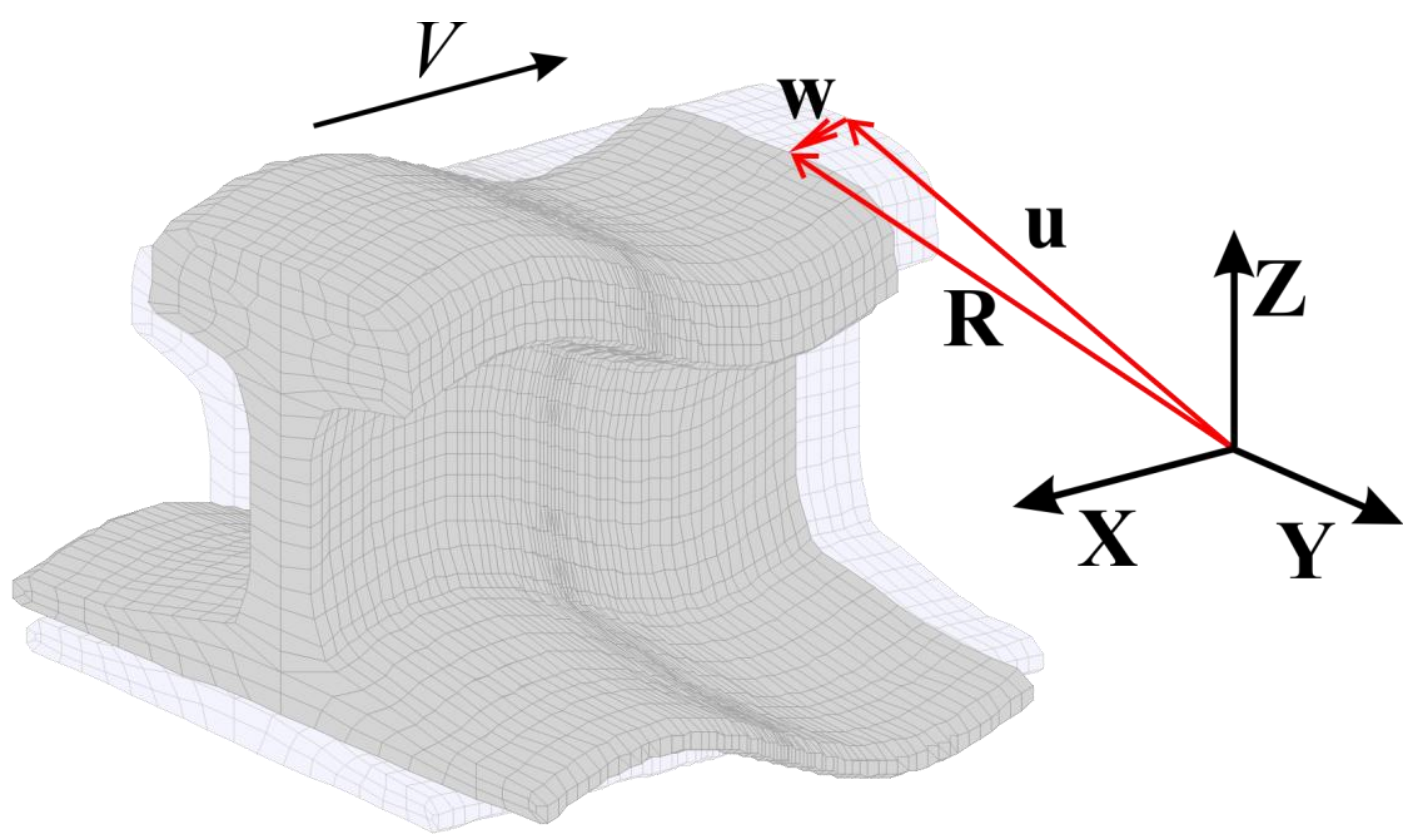

Figure 2: Finite element mesh of the rail (X-axis is out of scale). Deformed and undeformed configuration and coordinates.

Authors: J. Giner-Navarro, J. Martínez-Casas, F. D. Denia and L. Baeza. 


\section{FIGURE 3}

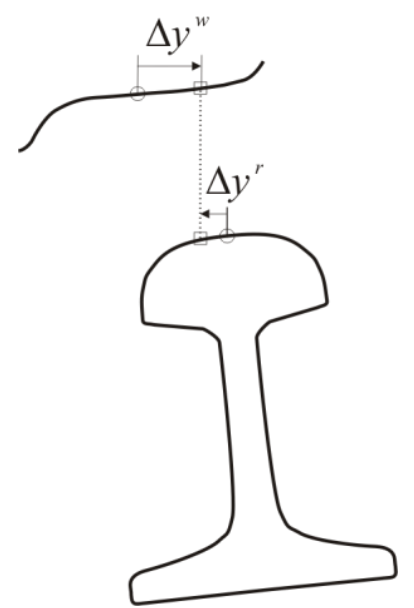

Figure 3: Lateral displacements of the wheel/rail contact point $\square$ with respect to the nominal one $\circ$ (for straight conditions).

Authors: J. Giner-Navarro, J. Martínez-Casas, F. D. Denia and L. Baeza. 
FIGURE 4 (Monochrome printers)

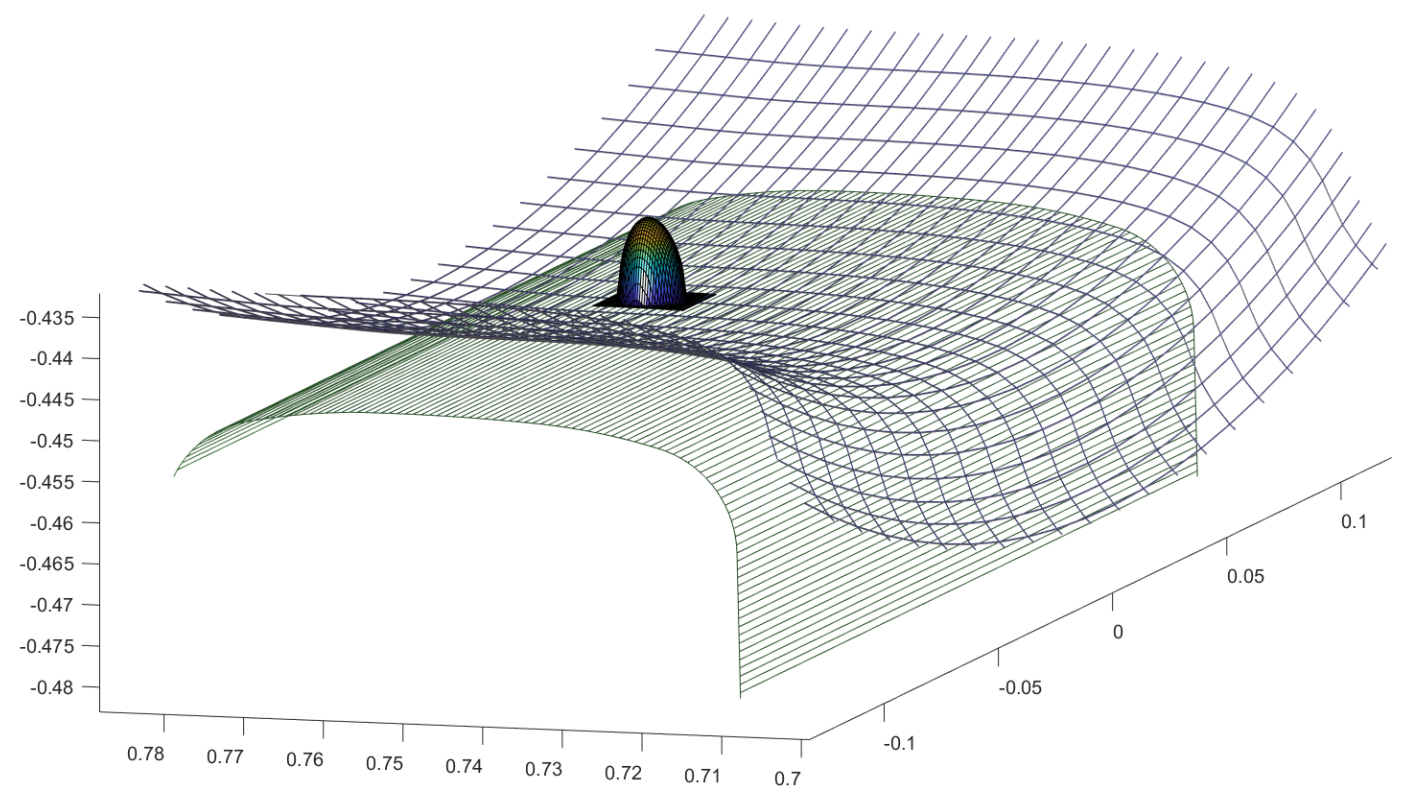

(a)

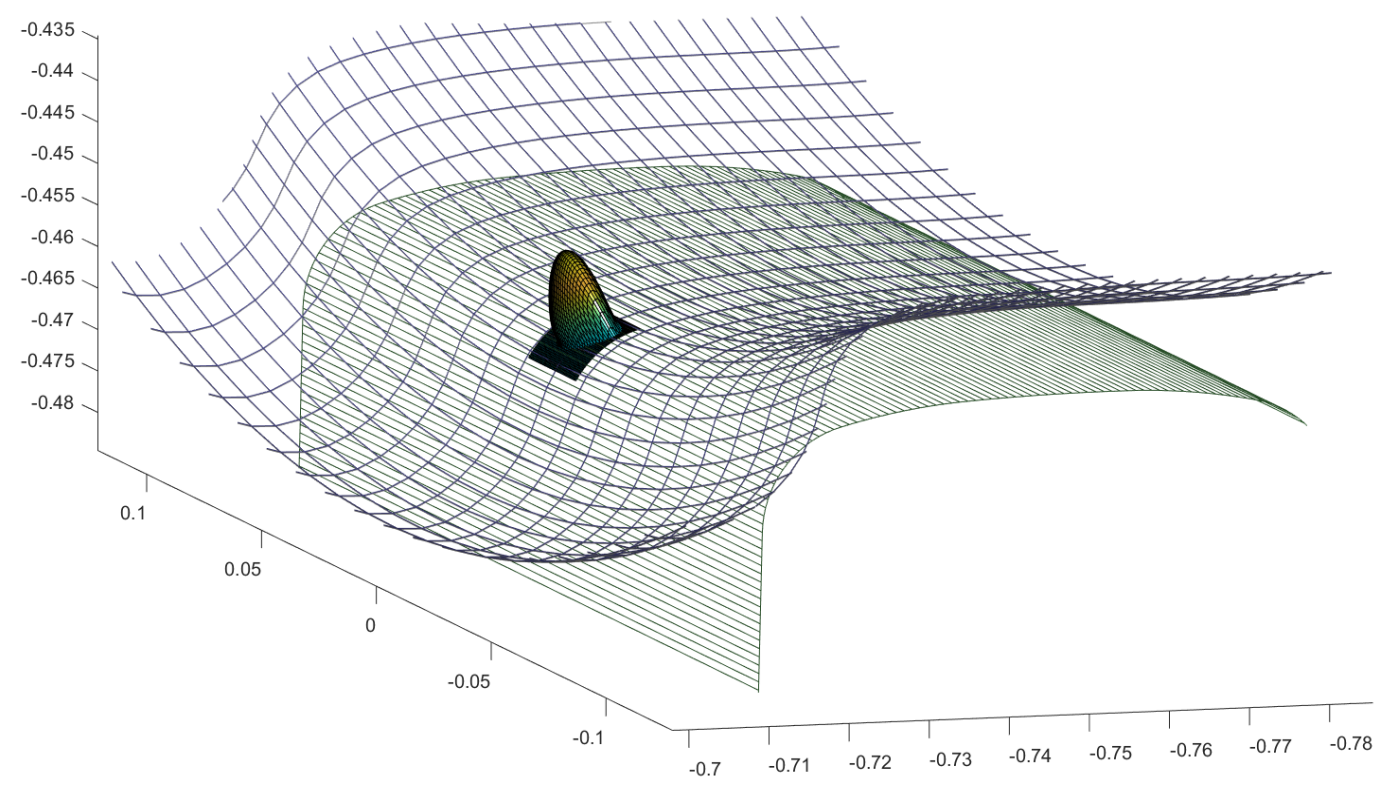

(b)

Figure 4: Three-dimensional view of the wheels (mesh) on the rails (solid colour). The locations of the wheel/rail contact (axes in $\mathbf{m}$ ) are shown by means of their normal traction distributions. The lateral displacement of the wheelset is $y=-6.2 \mathrm{~mm}$ and the yaw angle is $\psi=-0.264^{\circ}$ (quasi-static position of the wheelset during the curve negotiation), corresponding to Simulation I. (a) Inner to the curve wheel/rail pair; (b) outer to the curve wheel/rail pair.

Authors: J. Giner-Navarro, J. Martínez-Casas, F. D. Denia and L. Baeza. 
FIGURE 4 (Online version)

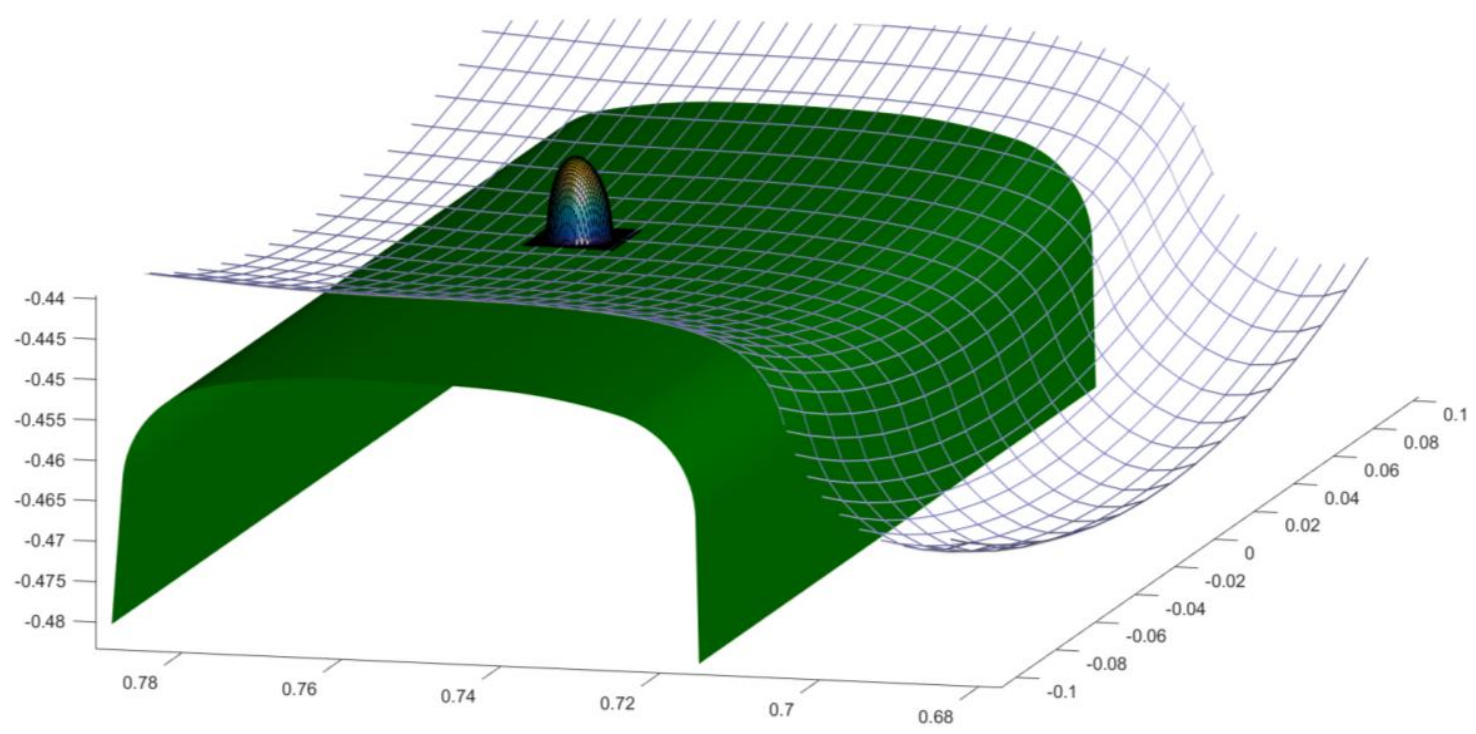

(a)

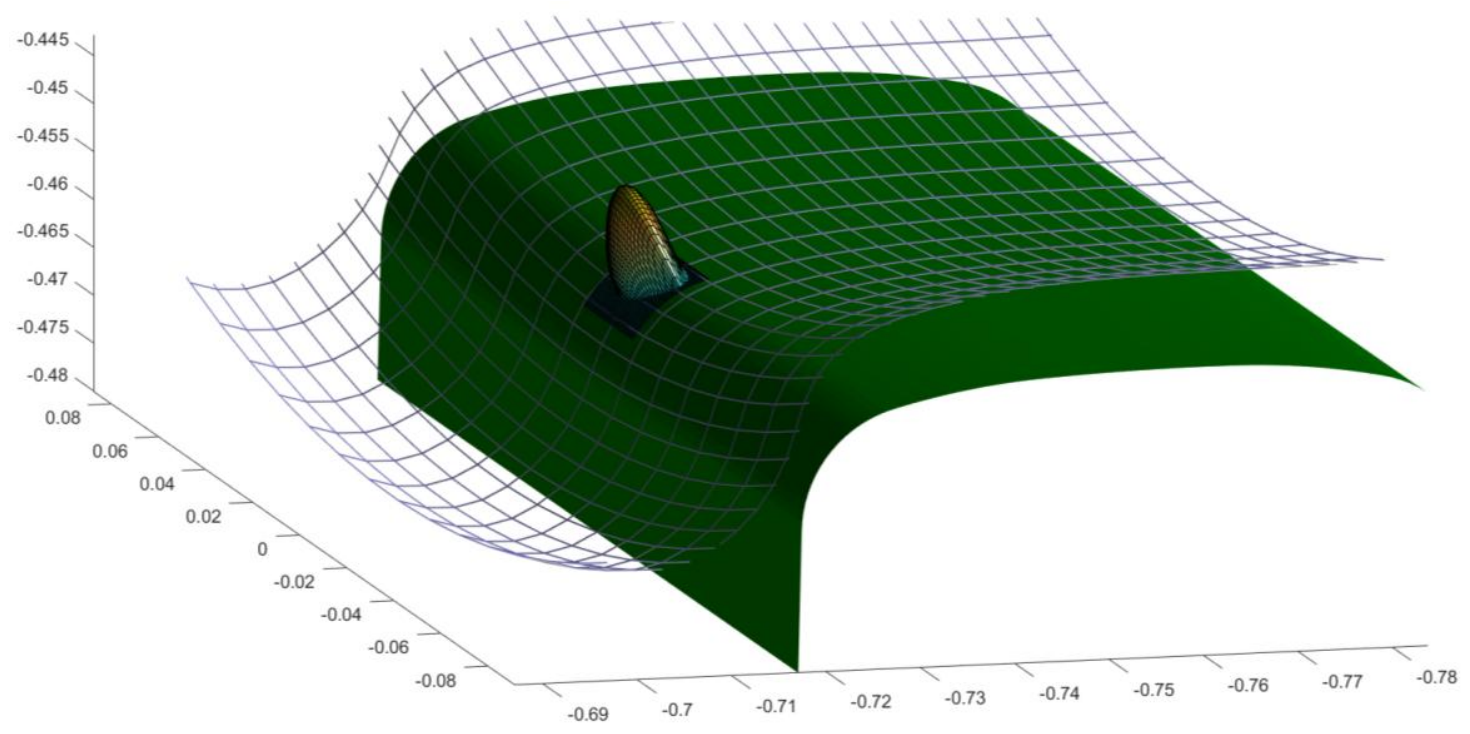

(b)

Figure 4: Three-dimensional view of the wheels (mesh) on the rails (solid colour). The locations of the wheel/rail contact (axes in $\mathbf{m}$ ) are shown by means of their normal traction distributions. The lateral displacement of the wheelset is $y=-6.2 \mathrm{~mm}$ and the yaw angle is $\psi=-0.264^{\circ}$ (quasi-static position of the wheelset during the curve negotiation), corresponding to Simulation I. (a) Inner to the curve wheel/rail pair; (b) outer to the curve wheel/rail pair.

Authors: J. Giner-Navarro, J. Martínez-Casas, F. D. Denia and L. Baeza. 


\section{FIGURE 5}

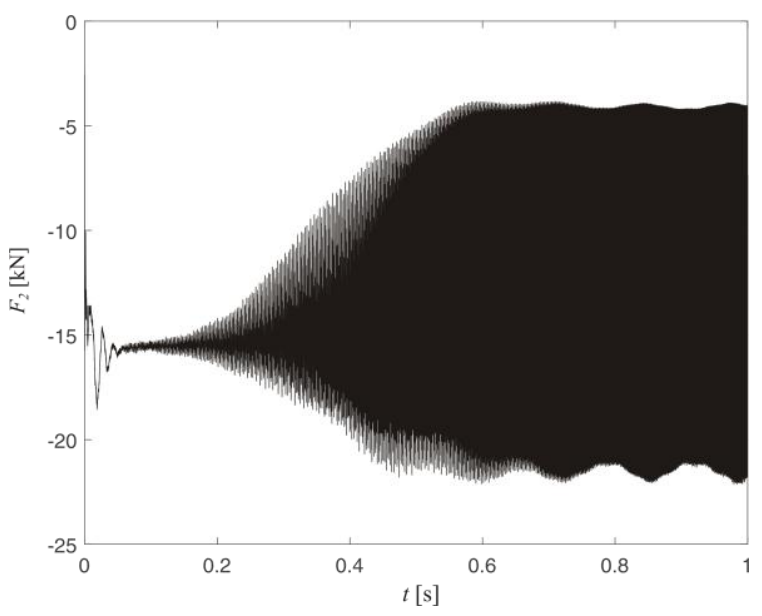

(a)

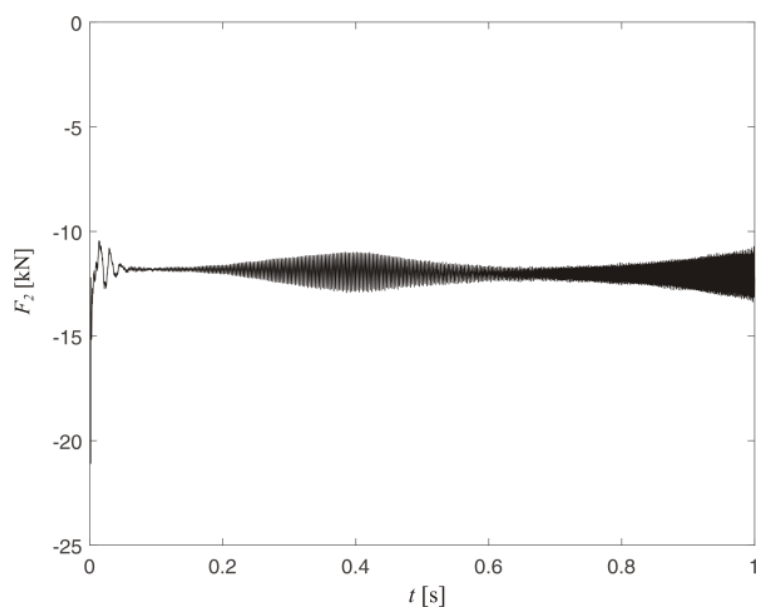

(b)

Figure 5: Time series of the tangential contact force $F_{2}$ corresponding to Simulation I for the inner (a) and outer (b) wheels.

Authors: J. Giner-Navarro, J. Martínez-Casas, F. D. Denia and L. Baeza. 
FIGURE 6

(a)

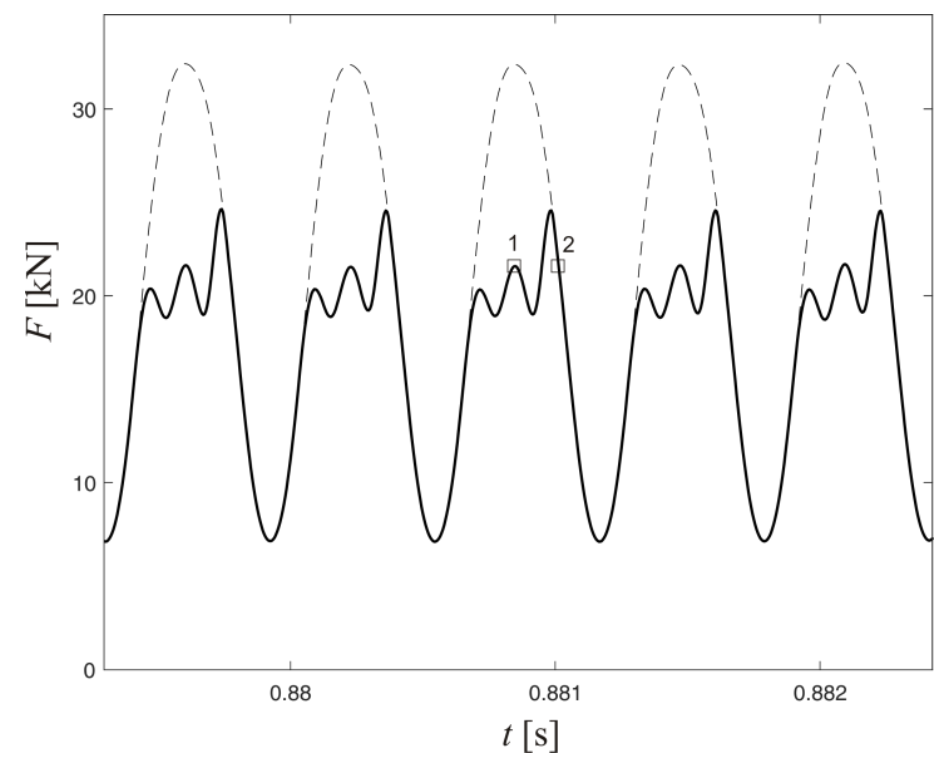

(b)

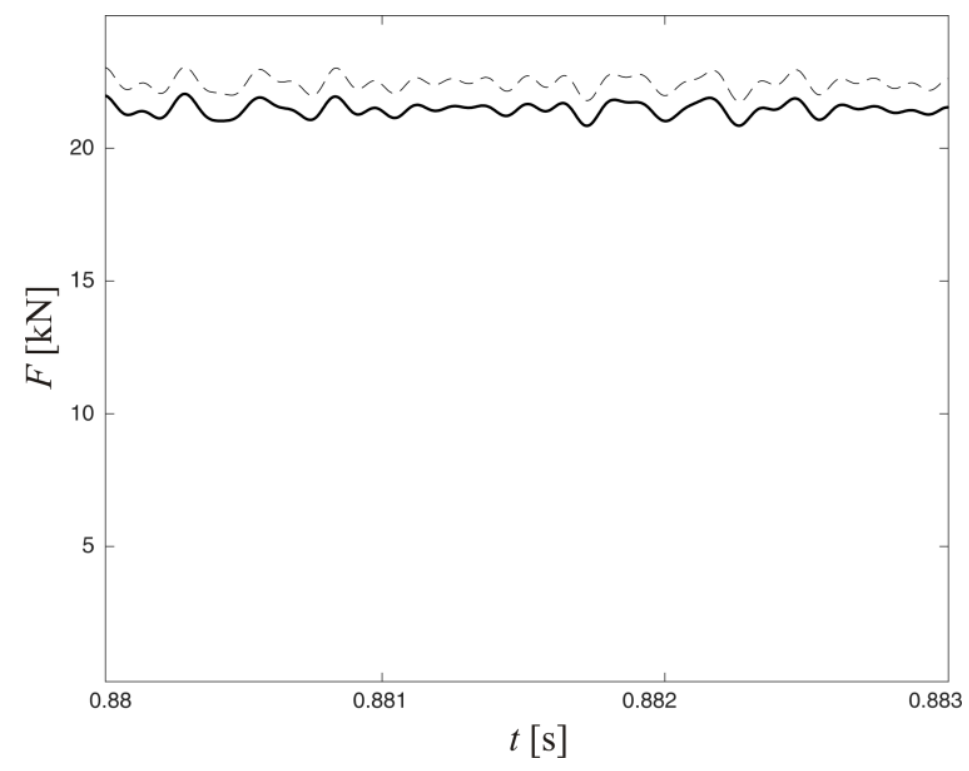

Figure 6: Zoom on time series of the contact forces; tangential force $F(-)$, traction bound $\mu F_{3}$ (- - -); some selected steps are marked with Arabic numerals. Simulation I: inner (a) and outer (b) wheels.

Authors: J. Giner-Navarro, J. Martínez-Casas, F. D. Denia and L. Baeza. 


\section{FIGURE 7}

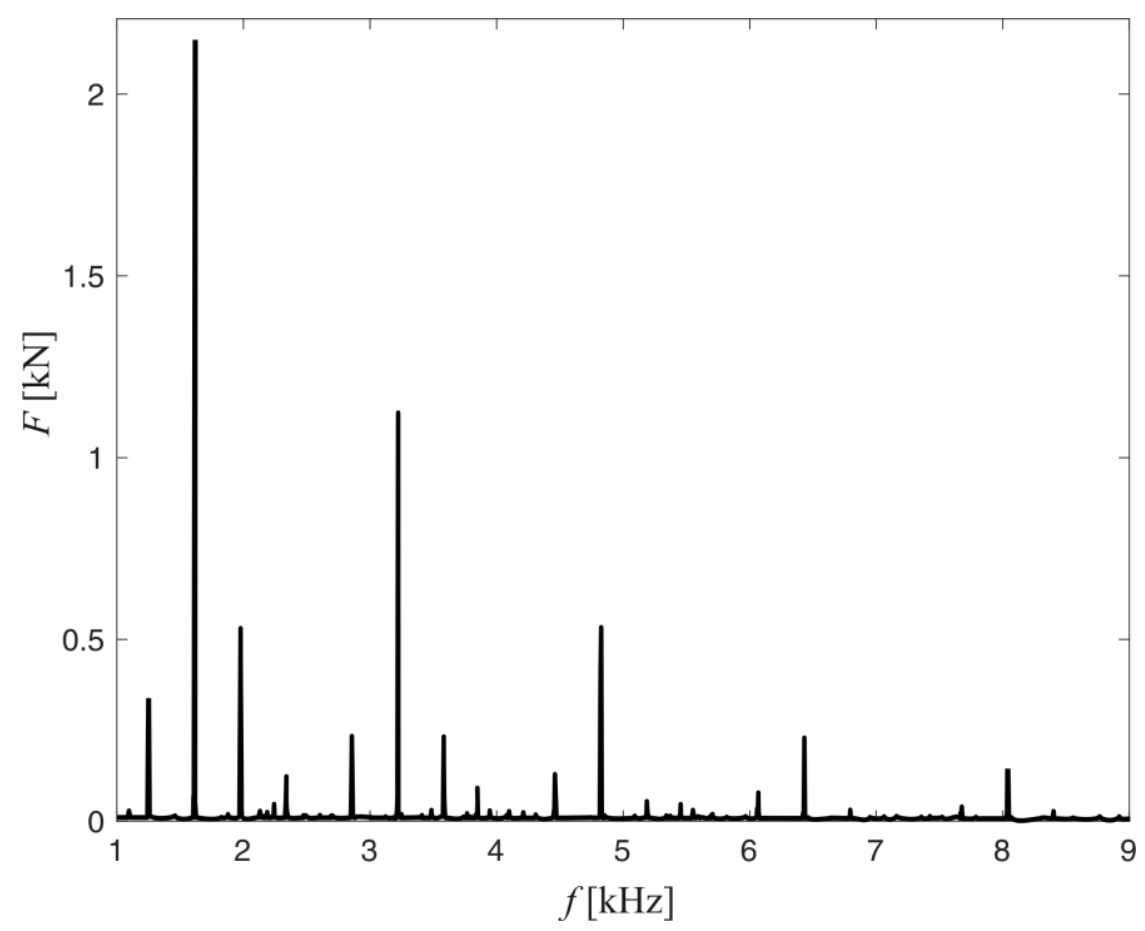

Figure 7: Frequency spectrum of the tangential contact force corresponding to Simulation I.

Authors: J. Giner-Navarro, J. Martínez-Casas, F. D. Denia and L. Baeza. 


\section{FIGURE 8}

(a)

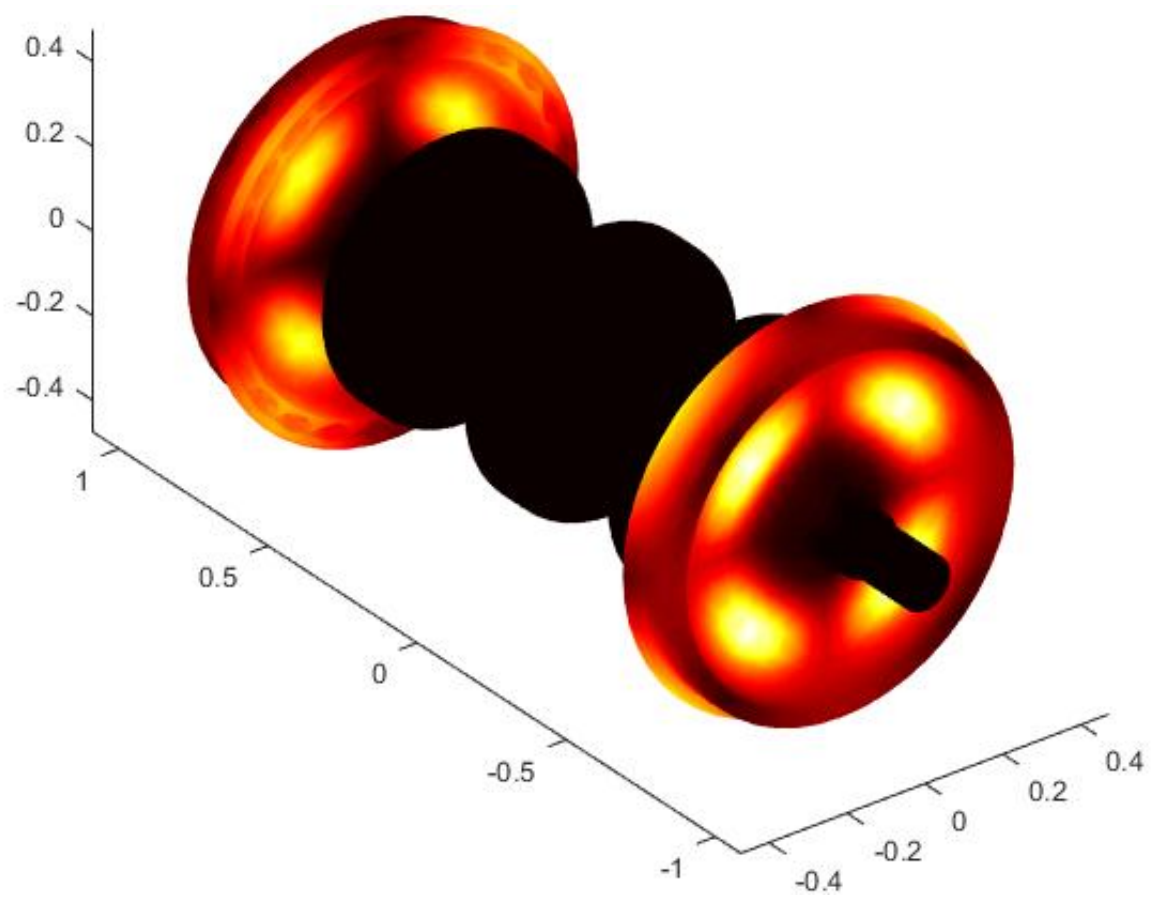

(b)

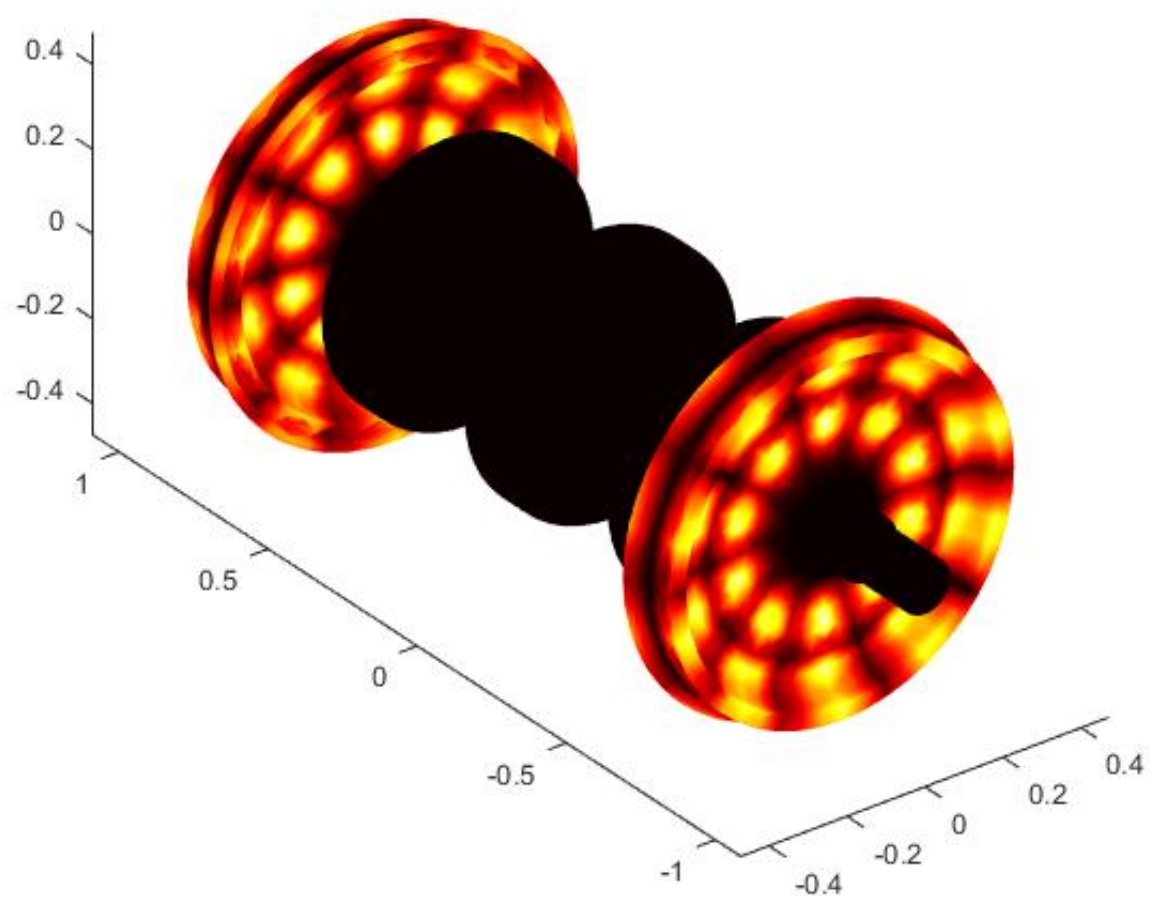

Figure 8: Mode shapes of the wheelset. (a) Axial $(2,0, a)$ wheel mode corresponding to $1607 \mathrm{~Hz}$;

(b) axial $(5,1, a)$ wheel mode corresponding to $4819 \mathrm{~Hz}$.

Authors: J. Giner-Navarro, J. Martínez-Casas, F. D. Denia and L. Baeza. 
FIGURE 9

(a)

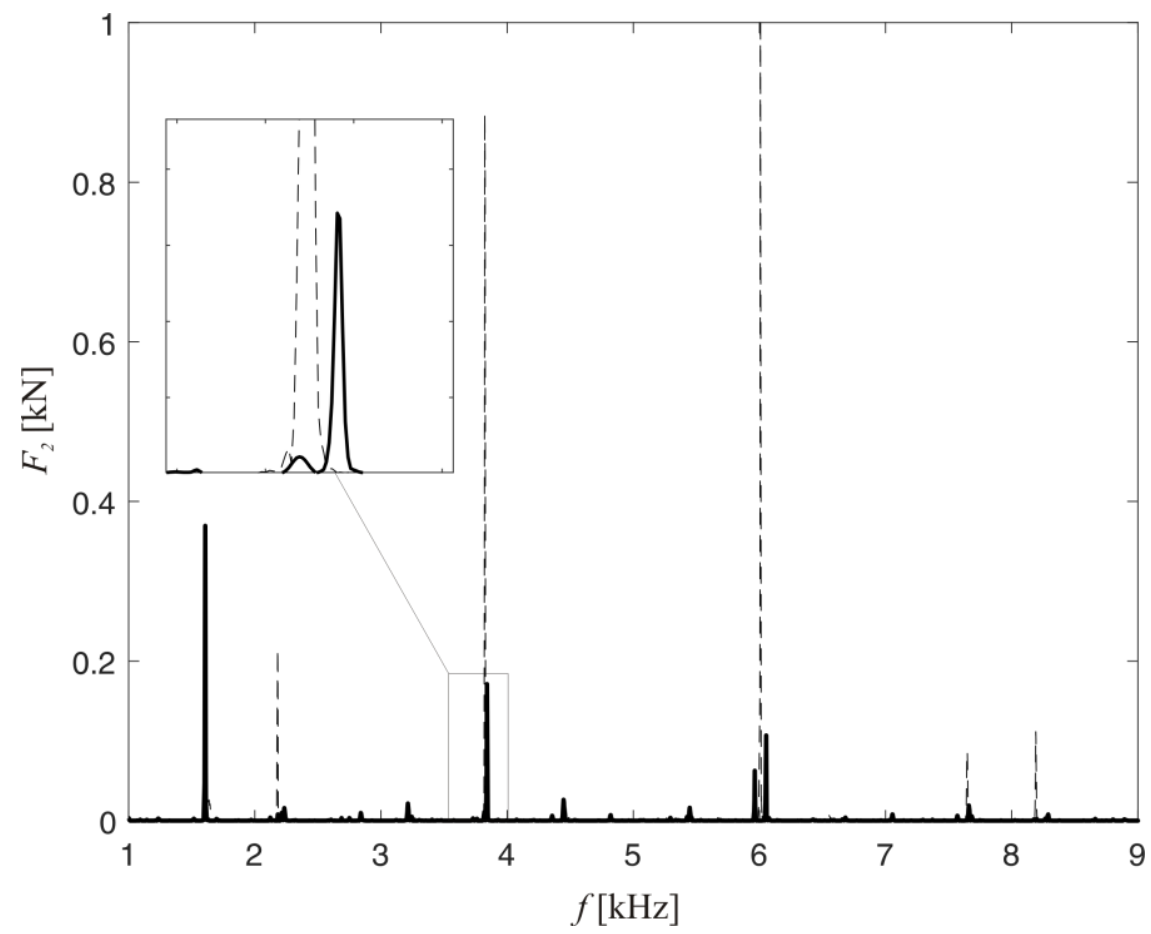

(b)

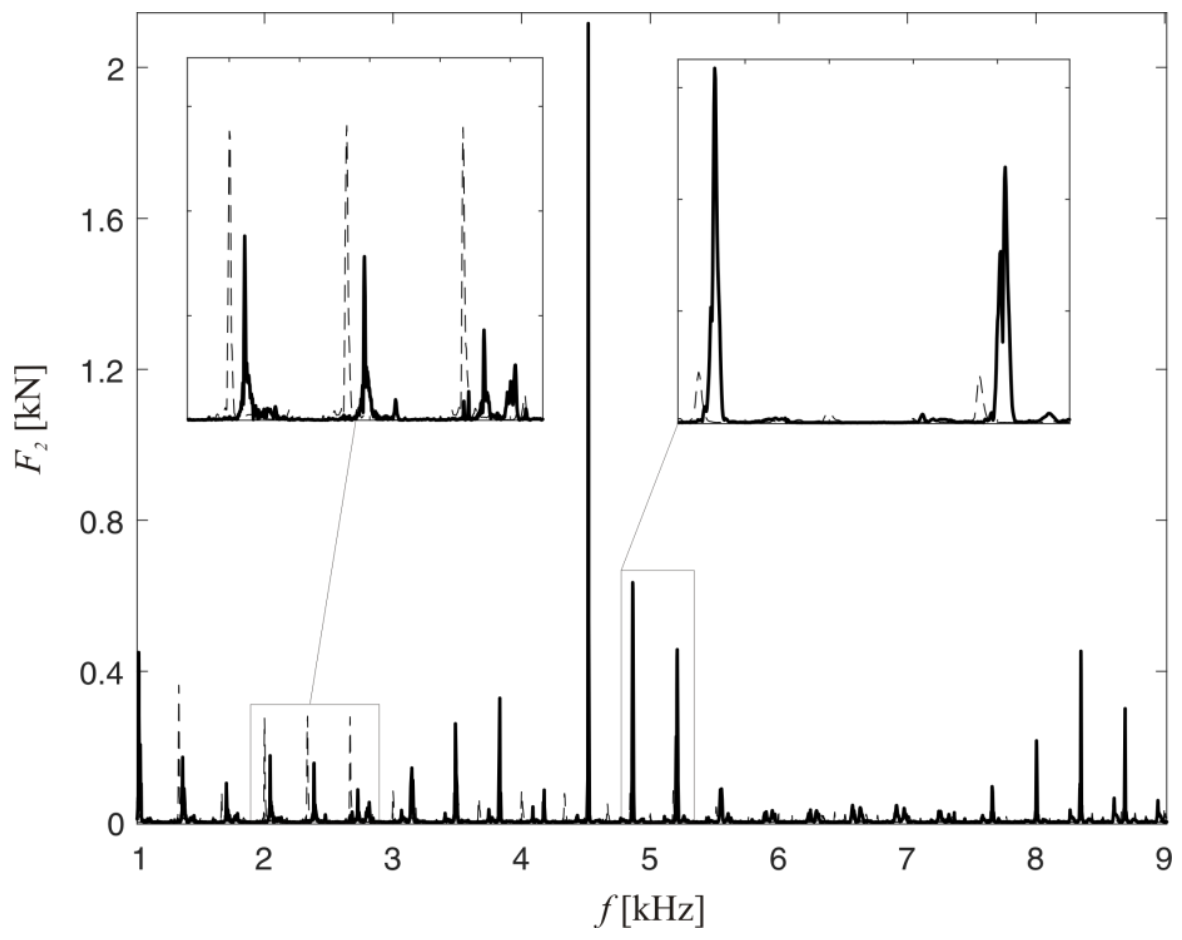


(c)

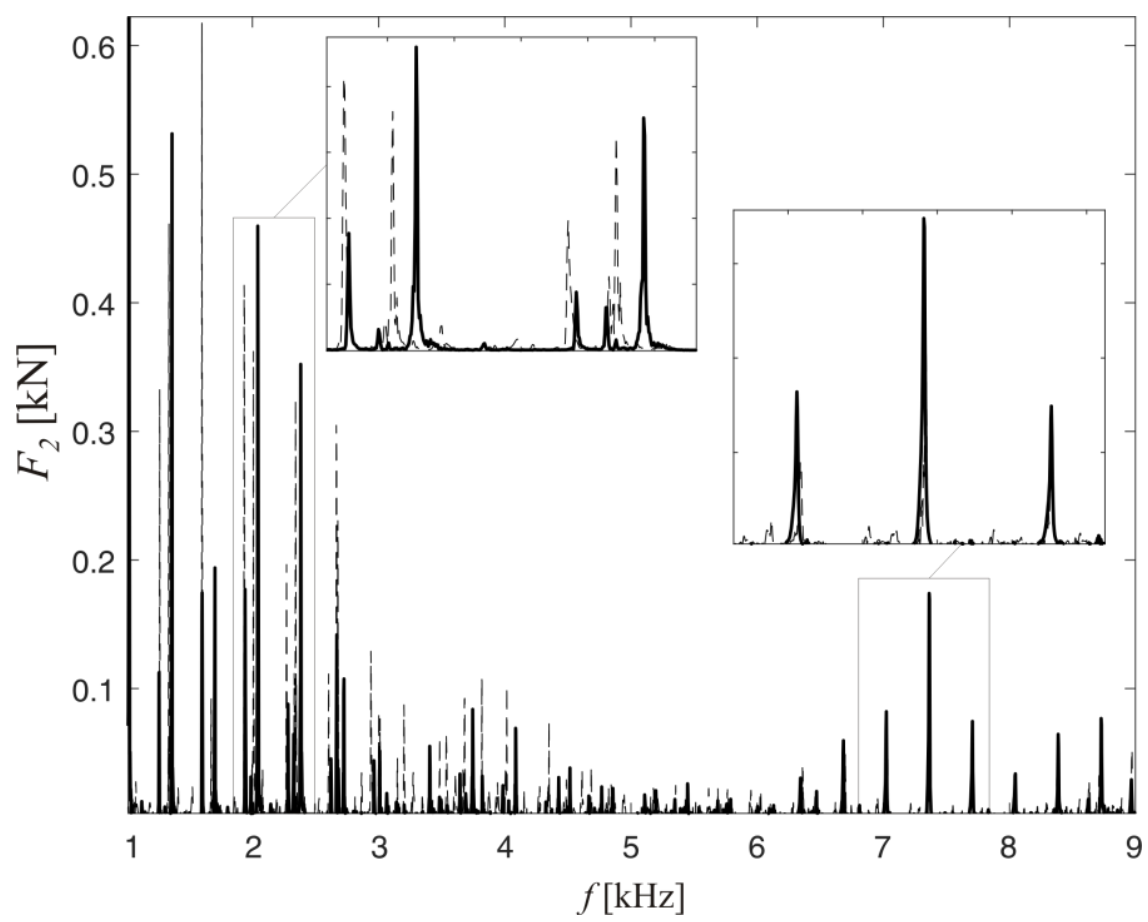

Figure 9: Frequency spectrum of the lateral contact force of the inner wheel using a rotatory

wheelset model (-), and a non-rotatory one (- - -) for Simulations II (a), III (b), IV (c).

Authors: J. Giner-Navarro, J. Martínez-Casas, F. D. Denia and L. Baeza. 


\section{FIGURE CAPTIONS}

Figure 1: Reference frames and position vectors. The undeformed configuration of the wheelset is shown in dashed trace; a generic position of the flexible wheelset is sketched in solid colours.

Figure 2: Finite element mesh of the rail (X-axis is out of scale). Deformed and undeformed configuration and coordinates.

Figure 3: Lateral displacements of the wheel/rail contact point $\square$ with respect to the nominal one $\circ$ (for straight conditions).

Figure 4: Three-dimensional view of the wheels (mesh) on the rails (solid colour). The locations of the wheel/rail contact (axes in $\mathrm{m}$ ) are shown by means of their normal traction distributions. The lateral displacement of the wheelset is $y=-6.2 \mathrm{~mm}$ and the yaw angle is $\psi=-0.264^{\circ}$ (quasi-static position of the wheelset during the curve negotiation), corresponding to Simulation I. (a) Inner to the curve wheel/rail pair; (b) outer to the curve wheel/rail pair.

Figure 5: Time series of the tangential contact force $F_{2}$ corresponding to Simulation I for the inner (a) and outer (b) wheels.

Figure 6: Zoom on time series of the contact forces; tangential force $F(-)$, traction bound $\mu F_{3}(--)$; some selected steps are marked with Arabic numerals. Simulation I: inner (a) and outer (b) wheels.

Figure 7: Frequency spectrum of the tangential contact force corresponding to Simulation I.

Figure 8: Mode shapes of the wheelset. (a) Axial (2,0,a) wheel mode corresponding to 1607 $\mathrm{Hz}$; (b) axial $(5,1, \mathrm{a})$ wheel mode corresponding to $4819 \mathrm{~Hz}$.

Figure 9: Frequency spectrum of the lateral contact force of the inner wheel using a rotatory wheelset model (-), and a non-rotatory one (- - -) for Simulations II (a), III (b), IV (c). 


\section{TABLE 1}

Table 1: Study cases simulated by the complete wheelset/track interaction model proposed.

\begin{tabular}{llll}
\hline Simulation & $\begin{array}{l}\text { Curve radius, } \\
R^{r}[\mathrm{~m}]\end{array}$ & $\begin{array}{l}\text { Vehicle speed, } \\
V[\mathrm{~km} / \mathrm{h}]\end{array}$ & $\begin{array}{l}\text { Friction coefficient, } \\
\mu[-]\end{array}$ \\
\hline V & 120 & 39.13 & 0.20 \\
VI & & & 0.32 \\
III & & & 0.40 \\
IV & & & 0.60 \\
\hline VII & 500 & 79.86 & 0.20 \\
II & & & 0.32 \\
I & & & 0.40 \\
VIII & & & 0.60 \\
\hline
\end{tabular}


TABLE 2

Table 2: Pseudo-static lateral variations of the position of the contact point (with respect to the nominal contact point) and creepages for each simulation case. The $\mathrm{Y}$-axis points forward the centre of the curve as illustrated in Fig. 1.

\begin{tabular}{|c|c|c|c|c|}
\hline Simulation & $\begin{array}{l}\text { Lat. variation contact } \\
\text { point inner wheel, } \\
\Delta y_{i n n}^{w}[\mathrm{~mm}]\end{array}$ & $\begin{array}{l}\text { Lat. variation contact } \\
\text { point outer wheel, } \\
\Delta y_{\text {out }}^{w}[\mathrm{~mm}]\end{array}$ & $\begin{array}{l}\text { Lat. creepage } \\
\text { inner wheel, } \\
\bar{\xi}_{2, \text { inn }}[-]\end{array}$ & $\begin{array}{l}\text { Lat. creepage } \\
\text { outer wheel, } \\
\bar{\xi}_{2, \text { out }}[-]\end{array}$ \\
\hline I & 14.3 & 33.3 & 0.0046 & 0.0055 \\
\hline II & 14.3 & 32.5 & 0.0048 & 0.0055 \\
\hline III & 14.5 & 36.2 & 0.0171 & 0.0268 \\
\hline IV & 14.5 & 38.3 & 0.0172 & 0.0437 \\
\hline V & 14.4 & 32.8 & 0.0169 & 0.0194 \\
\hline VI & 14.5 & 35.1 & 0.0171 & 0.0234 \\
\hline VII & 14.0 & 30.7 & 0.0052 & 0.0056 \\
\hline VIII & 14.4 & 34.6 & 0.0046 & 0.0056 \\
\hline
\end{tabular}


TABLE 3

Table 3: Simulation I: Quasi-static conditions for the leading wheelset.

\begin{tabular}{ll}
\hline Lateral displacement, $y[\mathrm{~mm}]$ & -6.2 \\
Vertical displacement, $z[\mathrm{~mm}]$ & 0.4 \\
Angle of attack, $\psi\left[{ }^{\circ}\right]$ & -0.264 \\
Longitudinal force, $\bar{X}[\mathrm{~N}]$ & 162 \\
Lateral force, $\bar{Y}[\mathrm{~N}]$ & -1770 \\
Vertical force, $\bar{Z}[\mathrm{~N}]$ & -109620 \\
\hline
\end{tabular}


TABLE 4

Table 4: Simulation I: Quasi-static conditions for the inner/outer wheels of the leading wheelset.

\begin{tabular}{ll}
\hline Rail lateral displacement, $\Delta y^{r}[\mathrm{~mm}]$ & $4.8 / 29.8$ \\
Rolling radius, $r_{11}[\mathrm{~mm}]$ & $459.7 / 465.6$ \\
Normal angle, $\theta\left[^{\circ}\right]$ & $0.56 / 33.40$ \\
Longitudinal creepage, $\bar{\xi}_{1}[-]$ & $0.0037 / 0.0059$ \\
Lateral creepage, $\bar{\xi}_{2}[-]$ & $0.0046 / 0.0055$ \\
Spin creepage, $\bar{\xi}_{s p}[-]$ & $0.021 / 1.185$ \\
Yaw angle, $\bar{\alpha}\left[^{\circ}\right]$ & 0.264 \\
Longitudinal creep force, $\bar{F}_{1}[\mathrm{~N}]$ & $13672 /-13510$ \\
Lateral creep force, $\bar{F}_{2}[\mathrm{~N}]$ & $15443 /-17213$ \\
Vertical creep force, $\bar{F}_{3}[\mathrm{~N}]$ & $-52072 /-57548$ \\
\hline
\end{tabular}




\section{TABLE 5}

Table 5: Main squeal frequencies in $\mathrm{kHz}$ (in bold for the main peak and in brackets for the corresponding amplitude in $\mathrm{kN}$ ) of the lateral contact forces of the inner wheel for the eight simulations carried out.

\begin{tabular}{l|llll}
\hline & $\mu=0.20$ & $\mu=0.32$ & $\mu=0.40$ & $\mu=0.60$ \\
\hline$R^{r}=120 \mathrm{~m}$ & $6.8, \mathbf{7 . 4}_{(1.2)}, 8.0$ & $1.4, \mathbf{4 . 5}_{(1.1)}$ & $\mathbf{4 . 5}_{(2.1)}, 8.0$ & $1.4,1.6,2.2, \mathbf{7 . 4}_{(0.5)}$ \\
$R^{r}=500 \mathrm{~m}$ & $\mathbf{3 . 8}_{(1.0)}, 7.7$ & $\mathbf{1 . 6}_{(1.4)}, 3.8,6.1,7.7$ & $\mathbf{1 . 6}_{(1.7)}, 3.2,4.8,6.4$ & $\mathbf{1 . 6}_{(2.2)}, 3.2,4.8,6.4$ \\
\hline
\end{tabular}


TABLE 6

Table 6: Closest wheelset modes associated with the main squeal frequencies for the inner wheel. Frequencies in bold corresponds exactly to wheelset modes.

\begin{tabular}{lll}
\hline Frequency [Hz] & Closest wheelset modes $(\mathbf{n}, \mathbf{m}, \mathbf{a} / \mathbf{r})$ & Simulation \\
\hline $\mathbf{1 3 6 1}$ & Axial $(0,0, a)$ wheel mode & VI \\
$\mathbf{1 6 0 7}$ & Axial $(2,0, a)$ wheel mode & I, II \\
$\mathbf{3 2 1 3}$ & Axial $(4,0, a)$ wheel mode & I, VIII \\
$\mathbf{3 8 4 1}$ & Radial axle mode & II, VII \\
4518 & Axial axle mode $(4545 \mathrm{~Hz})$ & III, VI \\
$\mathbf{4 8 1 9}$ & Axial $(5,1, a)$ wheel mode & I, VIII \\
6053 & Radial $(4,0, r)$ wheel mode $(6078 \mathrm{~Hz})$ & II \\
$\mathbf{6 4 2 5}$ & Axial axle mode & I, VIII \\
7449 & Combined mode $(7425 \mathrm{~Hz})$ & IIV, V \\
7680 & Axial axle mode & II, VII \\
$\mathbf{7 9 5 8}$ & Combined mode $(7980 \mathrm{~Hz})$ & III \\
\hline
\end{tabular}




\section{TABLE CAPTIONS}

Table 1: Study cases simulated by the complete wheelset/track interaction model proposed.

Table 2: Pseudo-static lateral variations of the position of the contact point (with respect to the nominal contact point) and creepages for each simulation case. The Y-axis points forward the centre of the curve as illustrated in Fig. 1.

Table 3: Simulation I: Quasi-static conditions for the leading wheelset.

Table 4: Simulation I: Quasi-static conditions for the inner/outer wheels of the leading wheelset.

Table 5: Main squeal frequencies in $\mathrm{kHz}$ (in bold for the main peak and in brackets for the corresponding amplitude in $\mathrm{kN}$ ) of the lateral contact forces of the inner wheel for the eight simulations carried out.

Table 6: Closest wheelset modes associated with the main squeal frequencies for the inner wheel. Frequencies in bold corresponds exactly to wheelset modes. 